\title{
On the competition of overstability and stabilizing effects in viscoelastic thermovibrational flow
}

\author{
Alessio Boaro and Marcello Lappe* \\ Department of Mechanical and Aerospace Engineering, University of Strathclyde, \\ James Weir Building, 75 Montrose Street, Glasgow, G1 1XJ, UK.
}

(Dated: July 21, 2021)

\begin{abstract}
Attention is paid to a specific form of thermal convection which encompasses viscoelastic and thermovibrational effects in a single problem or framework. The main objective is understanding the relationship between the phenomenon of overstability and periodic forcing through numerical solution of the governing equations in their complete, time-dependent and non-linear form. Fluid motion is found for values of the control parameter one order of magnitude smaller than the threshold to be exceeded in the equivalent Newtonian case. When the disturbances saturate their amplitude, patterns emerge that are reminiscent of the superlattice structures typical of "complex order". In the present case, such peculiar modes of convection are driven by the coexistence of two distinct spatial scales, each displaying a different temporal dependence, driven by the interplay of the timevarying (stabilizing/destabilizing) acceleration induced by vibrations and the ability of the fluid to store and release elastic energy.
\end{abstract}

\section{INTRODUCTION}

Thermal convection driven by different forces is omnipresent in nature and technology ([1-12).Despite the significant amount of results available for Newtonian fluids, however, the finite-amplitude states of this kind of flows in viscoelastic liquids have so far resisted an extensive and exhaustive analysis due to their inherent complexity and the intrinsic difficulties associated with the solution of the governing equations (well-known problems resulting from the existence of mathematical singularities which can jeopardize the stability of numerical algorithms [13 18]).

Most of the current knowledge is due to the linear stability analysis (LSA) approach. By virtue of these valuable studies, it is known that the presence of elasticity can determine onset of convection from an initially quiescent state uniformly heated from below for values of the governing parameter (the so-called Rayleigh number) smaller than that needed to induce fluid motion in an equivalent Newtonian medium. It is also known that such modes of convection are generally oscillatory (as opposed to the intrinsically stationary nature of standard Rayleigh-Bénard convection in non-elastic fluids). The existence of these two distinct classes of solutions simply reflects two fundamental instability mechanisms that can be enabled to produce convection from a motionless state: namely, either the so-called "exchange of stabilities", where the sign of a real stability exponent changes from negative to positive as the control parameter is increased (just as in the Newtonian case), or a concurrent process (known as "overstability"), where the real part of a pair of complex conjugate stability exponents becomes positive on passage through criticality.

Although the first LSA based results for the idealized situation of infinite layer date back to Green [19,

\footnotetext{
* marcello.lappa@strath.ac.uk
}

Vest and Arpaci 20], Sokolov and Tanner [21], numerical simulations for cavities with finite size, based on the direct solution of the overarching equations in their original (non-linear) form, have been produced much more recently. As outlined above, indeed, these problems are still a challenging task for numerical simulation and have instigated different possible strategies of attack; these efforts have led to disjoint glimpses of a broad range of qualitatively and quantitatively different results in widely disjoint sub-regions of the parameter space $([22,27][28$ 36. ).

This lack of consistently obtained results becomes even more evident if other types of thermally driven flows are considered, especially the variants of buoyancy convection where (unlike standard gravity) the driving force is not constant and varies in time. This is the case of the so-called thermovibrational convection (TV), i.e. fluid motion induced in a non-isothermal fluid by the application of vibrations (vibrating a closed cavity results in the fluid contained in it being subjected to an acceleration that changes periodically its sign with the same frequency of the vibrations [37). If such a container is considered in microgravity conditions, this is the only acceleration effectively producing convection ([2, 6, 7, 38]).

Unlike standard flows of thermogravitational nature (which can be found in a plethora of natural and industrial terrestrial processes), this specific form of fluid motion is extremely relevant to the area of space research; indeed, interest in it has sharply increased over recent years as a result of the advent of new orbiting platforms, which have made relatively long microgravity times available, and new technologies based on standard and complex fluids possible (see, e.g., 39 43]).

Thermovibrational convection can occur in Newtonian and viscoelastic liquids as well as in other complex fluids, thereby further expanding the variety of possible spatiotemporal phenomena. Notably, even if attention is limited to purely Newtonian fluids, modifications with respect to standard thermogravitational flow are substan- 
tial and concern both the structure of the flow and the related hierarchy of bifurcations [44, 45].

By virtue of existing studies for Newtonian fluids (2, 4, 6, 7, 40, 46, 54]), it has been clarified that in addition to the values of the acceleration and imposed temperature difference $\Delta T$ (contributing to the magnitude of the Rayleigh number), other important influential factors must be sought in the angle that the direction of shaking forms with the imposed temperature difference, and the frequency (and amplitude) of vibrations. In particular, the latter can be used to split the range of possible solutions into two main branches, namely, that of high frequency and small amplitude (also known as Gershuni's asymptotic regime, [38, 55, [59]) and, a second regime corresponding to small frequencies and high amplitudes. In the former, non-linear effects associated with the (Navier-Stokes and energy) equations governing fluid flow can enable a significant steady component of fluid velocity (which can typically be revealed by timeaveraging the velocity field), whereas in the latter, the time-dependent response of the fluid to the application of vibrations is dominant (the fluid moving with the same frequency of the vibrations as it were driven by a linear cause-and-effect relationship with the imposed forcing).

As shown by several investigators, if the asymptotic Gershuni regime is attained, vibrations perpendicular to the temperature gradient can produce interesting patterns 60 63, vice versa, their main effect is that of suppressing any form of convection if they are parallel to such a gradient 64. However, the outcomes become dramatically different when the complementary (opposite) regime with small frequencies is considered. In this case, a variety of interesting states become possible in Newtonian fluids if vibrations are parallel to the temperature difference ([65, 66]). Their complexity should essentially be regarded as a consequence of the interplay between specific destabilizing and stabilizing effects, which are established in these circumstances.

While an appreciable amount of knowledge has been produced for Newtonian fluids (as witnessed by the literature cited above), unfortunately, only a handful of results are available for viscoelastic liquids. As an example, in the attempt to shed some light on these behaviors, Lyubimova and Kovalevskaya [67] investigated the case of an infinite layer of viscoelastic liquid simultaneously subjected to a steady and a time-varying acceleration (the steady and oscillatory components having similar amplitude). Although different interesting new physical mechanisms were identified, the root dynamics driving these types of solutions are still largely unknown. In particular, no studies have appeared where the "pure" thermovibrational flow in viscoelastic fluids has been examined (with the only exception of that by Boaro and Lappa 68 where however, the focus was on the situation with vibrations perpendicular to the temperature difference for which overstability is not a relevant mechanism).

This automatically results in two important issues or

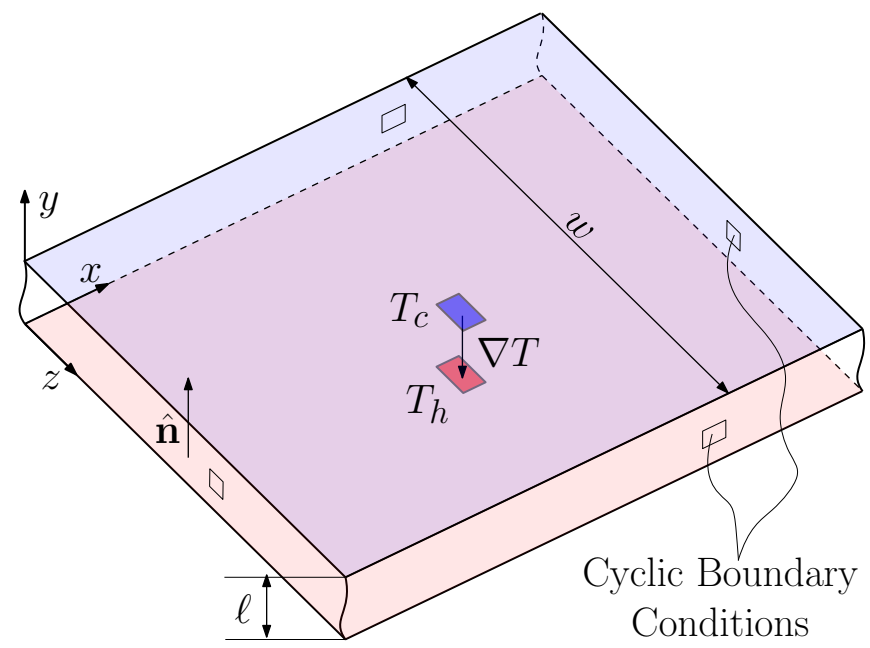

FIG. 1. Sketch of the geometry and scheme of the problem.

questions, which need to be pinpointed suitably here: is the overstability concept still applicable to pure thermovibrational convection? Moreover, it is unknown whether the mechanisms supposed to be operative in the case of Newtonian fluids still play a role in this case or not. Beyond the mere motivation to bridge the abovementioned gap, we are also specifically interested in pushing forward viscoelastic thermovibrational convection in a shallow cavity as a new archetypal problem to improve our fundamental understanding of some of the processes discussed before.

\section{MATHEMATICAL MODEL}

As discussed in the introduction, briefly stated, the problem is to determine how a relatively high level of elasticity can interfere with the low-frequency branch of thermovibrational convection when vibrations are parallel to the imposed temperature gradient.

From a mathematical point of view, for simplicity, we model this problem as a parallelepipedic shallow domain having a square symmetry (same size along the $x$ and $z$ directions) and aspect-ratio $A R=w / \ell=15$ (where $w$ represents the side of the bases and $\ell$ is the related distance along the y axis). The top and bottom boundaries are considered as solid no-slip walls (solid-solid configuration) having fixed temperature (Dirichlet boundary condition) in such a way that the difference of temperature $\Delta T=T_{h}-T_{c}$ is maintained constant. Moreover, in order to mimic the behaviour of and infinite layer, cyclic boundary conditions are implemented at the sides. A sketch of this configuration is shown in FIG. 1.

External vibrations are taken into account assuming that the displacement evolution follows a sinusoidal motion-law: 
where $b$ is the amplitude, $\omega$ is the angular frequency $(\omega=2 \pi f), \hat{\mathbf{n}}$ is the direction of the vibrations (in this work it is fixed and parallel to the temperature gradient $\nabla T$, as sketched in FIG. 1) and the symbol $\left({ }^{*}\right)$ states that the quantities are in dimensional form. The resulting acceleration, that is the second derivative of the displacement, is

$$
\mathbf{a}_{\omega}\left(t^{*}\right)=\gamma \sin \left(\omega t^{*}\right)
$$

where $\boldsymbol{\gamma}=-b \omega^{2} \hat{\mathbf{n}}$. This acceleration formally replaces the steady gravitational acceleration $\mathbf{g}$ in the buoyancy term derived with the Oberbeck-Boussinesq approximation.

At this stage, it is worth recalling that, unlike other forms of natural convection, historically, the investigation of this topic has taken a peculiar path of progression due to the discovery of the possibility to greatly simplify the inherent computational complexity. This potential simplification stems from the aforementioned ability of these flows to develop a dominant stationary response (in addition to the oscillatory one) in the limit of high frequency and small amplitude of the imposed vibrations. As a result of this peculiar property, alternate mathematical formulations (based on a "potential-flow" approach) have been developed, which are able to provide directly the time-averaged component of the velocity with no need to determine the associated fluctuating one (relevant examples of this modus operandi being the works by [38, 51, 55, 57]). Notably, several investigators could successfully use this strategy to analyze thermovibrational flow in cavities with different aspect ratio and vibrations orthogonal to the temperature gradient.

This formulation, however, is not suitable for the situation in which vibrations are parallel to the imposed temperature gradient. Moreover, it requires the flow to be in the Gershuni asymptotic regime (which would actually limit the investigation to the condition for which this approximation is satisfied).

For these reasons, the fluid motion balance equations, have been solved here in their original formulation, which in dimensional form reads:

$$
\begin{gathered}
\nabla \cdot \mathbf{u}^{*}=0 \\
\rho \frac{\partial \mathbf{u}^{*}}{\partial t^{*}}+\rho \nabla \cdot\left(\mathbf{u}^{*} \mathbf{u}^{*}\right)=-\nabla p^{*}+\eta_{s} \nabla^{2} \mathbf{u}^{*}+\nabla \cdot \tilde{\boldsymbol{\tau}}^{*} \\
+\rho \beta_{T}\left(T-T_{\mathrm{ref}}\right) \mathbf{a}_{\omega}\left(t^{*}\right) \\
\frac{\partial T^{*}}{\partial t^{*}}=-\nabla \cdot\left(\mathbf{u}^{*} T^{*}\right)+\alpha \nabla^{2} T^{*}
\end{gathered}
$$

where $t^{*}$ is the time, $\mathbf{u}^{*}$ is the velocity, $T^{*}$ is the temperature, $p^{*}$ is the pressure, $\rho$ is the density of the fluid, $\eta_{s}$ is the dynamic viscosity of the solvent (see the discussion reported later for additional information on this specific point; obviously, if a Newtonian fluid is considered, $\eta_{s}=\eta$, i.e. $\eta_{s}$ will account for the total viscosity of the fluid), $\beta_{T}$ is the thermal expansion coefficient and $\alpha$ is the thermal diffusivity. Eventually, $\tilde{\boldsymbol{\tau}}^{*}$ represent the extra-stress tensor due to the viscoelasticity of the fluid.

In order to get closure of this problem from a mathematical point of view, it is crucial to model $\tilde{\tau}^{*}$ as a function of other fluid-dynamic quantities. This relationship can be obtained using different rheological frameworks. In the present study, we adopt the well-known model based on the assumption that the viscoelastic fluid is a combination between a Newtonian solvent, having dynamic viscosity $\eta_{s}$, and a polymeric solute, having dynamic viscosity $\eta_{p}$. Moreover, with this approach, the polymeric part, which from a physical point of view consists of long polymeric chains, is schematized as two beads linked by a spring. This assumption is at the root of the so-called Dumbbell paradigm, able to describe adequately a class of liquid known as Boger fluid, i.e. highly elastic solutions that shows a constant viscosity over a wide range of shear rate. These fluids are characterized by a total dynamic viscosity $\eta_{0}=\eta_{s}+\eta_{p}$. Accordingly, two mutually dependent dimensionless groups related to the viscosity can be defined, i.e. the solvent-to-total viscosity ratio as $\xi=\eta_{s} / \eta_{0}$ and the viscosity ratio $\zeta=\eta_{p} / \eta_{s}$ $(\zeta=(1-\xi) / \xi)$.

There are several variants that have originated from the dumbbell paradigm, however, the Oldroyd-B model, has been the most widespread archetype for the investigation of thermal convection (see e.g. [36, 69, 70]). Moreover, this framework has been used to tackle thermal convection with modulated gravity in the work by Lyubimova and Kovalevskaya [67. Therefore, to be consistent with the existing literature and be able to compare our three-dimensional (3D) results with previous twodimensional (2D) or LSA studies we intentionally rely on the Oldroyd-B paradigm for the investigation of the present problem. Accordingly, the transport equations of $\tilde{\tau}^{*}$ can be cast in compact dimensional form as

$$
\begin{aligned}
\lambda\left(\frac{\partial \tilde{\boldsymbol{\tau}}^{*}}{\partial t^{*}}+\mathbf{u}^{*} \cdot \nabla \tilde{\boldsymbol{\tau}}^{*}\right) & +\tilde{\boldsymbol{\tau}}^{*}=\eta_{p}\left(\nabla \mathbf{u}^{*}+\left(\nabla \mathbf{u}^{*}\right)^{\boldsymbol{\top}}\right) \\
& +\lambda\left(\tilde{\boldsymbol{\tau}}^{*} \cdot \nabla \mathbf{u}^{*}+\left(\nabla \mathbf{u}^{*}\right)^{\boldsymbol{\top}} \cdot \tilde{\boldsymbol{\tau}}^{*}\right)(6
\end{aligned}
$$

where $\lambda$ is the relaxation time.

The non-dimensional form of eqs. (3), (4), (5) and (6) is obtained in the present work scaling the length with the depth $\ell$ of the layer, the velocity with $\alpha / \ell$, the time with $\ell^{2} / \alpha$, the frequency with $\alpha / \ell^{2}$, the pressure with $\rho \alpha^{2} / \ell^{2}$, the temperature with $\Delta T=T_{h}-T_{c}$ and the extra-stress tensor $\tilde{\boldsymbol{\tau}}^{*}$ with $\rho \nu_{s} \alpha / \ell^{2}$. Here, $\nu_{s}$ is the kinematic viscosity of the Newtonian solvent $\left(\nu_{s}=\eta_{s} / \rho\right)$. Therefore, the non-dimensional balance equations in their timedependent non-linear form read: 


$$
\begin{gathered}
\nabla \cdot \mathbf{u}=0 \\
\frac{\partial \mathbf{u}}{\partial t}=-\nabla p-\nabla \cdot(\mathbf{u u})+\operatorname{Pr} \nabla^{2} \mathbf{u}+\operatorname{Pr} \nabla \cdot \tilde{\boldsymbol{\tau}} \\
-P r_{g} R a_{\omega} T \sin (\Omega t) \hat{\mathbf{n}} \\
\frac{\partial T}{\partial t}=-\nabla \cdot(\mathbf{u} T)+\nabla^{2} T \\
\left.\frac{\partial \tilde{\boldsymbol{\tau}}}{\partial t}+\mathbf{u} \cdot \nabla \tilde{\boldsymbol{\tau}}\right)+\tilde{\boldsymbol{\tau}}=\zeta\left(\nabla \mathbf{u}+(\nabla \mathbf{u})^{\boldsymbol{\top}}\right) \\
+\vartheta\left(\tilde{\boldsymbol{\tau}} \cdot \nabla \mathbf{u}+(\nabla \mathbf{u})^{\top} \cdot \tilde{\boldsymbol{\tau}}\right)
\end{gathered}
$$

As the Reader might have realized at this stage, putting the equations in this form, naturally leads to the introduction of some additional relevant non-dimensional numbers ( $\zeta$ and $\xi$ have been previously defined), namely, the Prandtl number for the Newtonian solvent $\mathrm{Pr}=$ $\nu_{s} / \alpha$, a generalized version of this parameter for the viscoelastic fluid $\operatorname{Pr}_{g}=\operatorname{Pr} / \xi$, the non-dimensional frequency $\Omega=\ell^{2} \omega / \alpha$, the elasticity number $\vartheta=\lambda \alpha / \ell^{2}$, and the vibrational Rayleigh number:

$$
R a_{\omega}=\frac{b \omega^{2} \beta_{T} \Delta T \ell^{3}}{\nu_{0} \alpha}
$$

where $\nu_{0}=\eta_{0} / \rho$ is the total kinematic viscosity. The Rayleigh number introduced here is a variant of the classical Rayleigh number traditionally used to characterize standard buoyancy convection. The expression is the essentially the same; however, the steady gravitational acceleration is replaced by the amplitude of the acceleration induced by the considered mono-chromatic frequency vibrations.

It is now worth recalling that the elasticity number (see [27, 36, 69]), just like the equivalent Deborah number (see e.g. [35]), represents the ratio between two characteristic times, i.e. the characteristic time of the polymer molecules dynamics $(\lambda)$ and the thermal diffusion time $\left(t_{\alpha}=\ell^{2} / \alpha\right)$. However, it should also be recalled that, for the case of thermovibrational convection, another time scale is involved, i.e. that of the considered external forcing: the non-dimensional frequency $\Omega$ can also be seen as a ratio between period of the oscillations $T_{\omega}^{*}=2 \pi / \omega$ and $t_{\alpha}$ up to a multiplicative constant $2 \pi$. Given these arguments and following the same rationale of [68], therefore, we also introduce $\Sigma$, i.e. the ratio of the relaxation time $\lambda$ and $T_{\omega}^{*}$ :

$$
\Sigma=\frac{\lambda}{T_{\omega}^{*}}=\frac{\vartheta \Omega}{2 \pi}
$$

Although these three non-dimensional times are interwoven and thus $\vartheta$ and $\Omega$ would be sufficient for a complete characterization of the problem, we will also use the parameter $\Sigma$ given its ability to account for the relative importance of other concurrent physical effects.

\section{NUMERICAL METHOD}

The numerical procedure used in this study relies on the discretization of eqs. (7), (8), (9) and $(10)$ over the computational domain through a segregated finite volume method (as available in openFOAM ${ }^{\circledR}$ ). In particular, the exploited algorithm ( $P I S O$ ) pertains to the general category of primitive-variables techniques originally introduced by [71-73] and improved over the years by many other researchers ([74-77] just to cite a few). Its acronym stands for "Pressure-Implicit with Splitting of Operators"; it solves the set of balance equations interpolating the variable on a co-located grid though the Rhie and Chow 78, scheme.

The reason why we have not used other methods such as the PIMPLE[79] (generally faster than the PISO) for the present simulations is the following: in this specific problem, the time-step is 'constrained' by two different aspects: (i) the frequency of the vibrations, (ii) the stability of the viscoelastic model (not related to the classical Courant number). The time-step must be sufficiently small to avoid aliasing with respect the external vibrations, and to guarantee stability of the viscoelastic model at the same time. For these reasons, the advantages associated with other computational variants (such as the PIMPLE) become irrelevant (while related drawbacks, such as the increased computational cost, would be retained).

For the practical implementation of the PISO approach, the diffusive and convective terms appearing in the different equations have been discretized here using a CDS (Central Difference) scheme accurate to the secondorder. However, the CDS has been replaced with the Minmod variant for the specific solution of eq. (8). This 'workaround' has been introduced to mitigate some of the known numerical difficulties related to viscoelastic fluids, and ensure stability of the numerical procedure over a wide range of parameters. Nonetheless, to further improve the algorithm stability, following the methodology of Favero et al. 80, eq. (8) has been implemented numerically as:

$$
\begin{aligned}
& \frac{\partial \mathbf{u}}{\partial t}+\nabla \cdot(\mathbf{u u})-\operatorname{Pr}(1+\zeta) \nabla^{2} \mathbf{u}= \\
& -\nabla p-\operatorname{Pr} \zeta \nabla^{2} \mathbf{u}+\operatorname{Pr} \nabla \cdot \tilde{\boldsymbol{\tau}}-\operatorname{Pr}_{g} R a_{\omega} T \sin (\Omega t)
\end{aligned}
$$

With such a strategy, generally referred to in the literature as DEVSS (Discrete Elastic-Viscous-Split-Stress) method (see e.g. [81]), an extra diffusive term $\operatorname{Pr} \zeta \nabla^{2} \mathbf{u}$ is added at the left and right hand sides of eq. (8). Hence, from a purely mathematical point of view, eq. (8) and 
13) are equivalent. Nevertheless, to increase appreciably the "ellipticity" of the momentum equation and therefore improve the numerical stability of the time-marching procedure, one extra term is discretized in an implicit way while the other is implemented explicitly. The beneficial stabilization stems from the fact that the different treatment of the right- and left-hand-side term produces a quantitatively negligible numerical diffusion, which however appreciably improves the robustness of the solver.

\section{A. Validation}

The scope of the validation process is to verify the ability of the numerical procedure to capture and predict the onset of relevant fluid dynamic instabilities. An extensive discussion of the several tests used to validate the solver described in the earlier section is available in [27, 68]. Good agreement with the existing literature was found for both isothermal-fluid benchmarks (where the emerging instabilities are entirely elastic in nature) and non-isothermal fluid conditions (Rayleigh-Bénard convection).

For the convenience of the Reader, here we limit ourselves to recalling (briefly) the outcomes of the comparison with the linear stability analysis by MartínezMardones and Pérez-García [70] for the onset of standard Rayleigh-Bénard convection in a layer of Oldroyd-B fluid with $\operatorname{Pr}_{g}=10, \xi=0.5$ and $\vartheta=0.1$. The LSA analysis estimates that the bifurcation from a quiescent to an unsteady state occurs at $R a=1700$ if solid-solid boundary condition are applied to the layer. This bifurcation is sub-critical (overstability) and the arising flow oscillates with an angular frequency $\tilde{\omega}=4.63$. Through $2 \mathrm{D}$ non-linear simulations and using a mesh having 4500 elements, we calculated the value of $\tilde{\omega}$ for different values of $R a$. Extrapolating with a quadratic law $\tilde{\omega}$ to $R a=1700$, we predicted a value of the angular frequency that differs by $\approx 2 \%$ from the one predicted by the linear stability analysis.

Given the range of values of the elasticity parameter considered in the present analysis, as an additional step of validation, we have considered additional comparison with the recent work (LSA) by Lyubimova and Kovalevskaya 67].

These authors focused on buoyancy convection in a horizontal layer of Oldroyd-B fluid with free-free boundary conditions subjected to vibrations (the problem being therefore equivalent to considering an acceleration $\mathbf{a}_{\omega}(t)$ added to the steady gravitational acceleration $\mathbf{g}$ ). Moreover, they used a square wave as external acceleration profile. Accordingly, for the present non-linear unsteady simulations, the acceleration has been modeled using the following function:

$$
\mathbf{a}_{\omega}\left(t^{*}\right)=\gamma \tanh \left(10 \sin \left(\omega t^{*}\right)\right)
$$

leading to rewrite the buoyancy term appearing in the momentum equation (in dimensional and in nondimensional form, respectively) as:

$$
\mathbf{B}\left(t^{*}\right)=\rho g \beta\left(T^{*}-T_{0}^{*}\right)\left(1+\frac{b \omega^{2}}{g} \tanh \left(10 \sin \left(\omega t^{*}\right)\right)\right) \hat{\mathbf{i}}_{g}
$$

and

$$
\mathbf{B}(t)=\operatorname{RaT}(1+\Gamma \tanh (10 \sin (\Omega t))) \hat{\mathbf{i}}_{g}
$$

where $R a$ is the classical Rayleigh number based on the steady gravitational acceleration, $\Gamma=b \omega^{2} / g$ is the non-dimensional amplitude of the oscillatory acceleration and $\hat{\mathbf{i}}_{g}$ is the direction of the gravitational acceleration. Moreover, the condition

$$
\left[\left((\nabla \mathbf{u})+(\nabla \mathbf{u})^{\top}\right)+\tilde{\boldsymbol{\tau}}\right] \cdot \hat{\mathbf{n}}_{\mathrm{B}}=0
$$

has been assumed for the top and bottom boundaries, where $\hat{\mathbf{n}}_{\mathrm{B}}$ is the unit vector perpendicular to the boundary.

For $\operatorname{Pr}_{g}=7, \Omega=26.5, \Gamma=1, \xi=0.1, \vartheta=0.38$, Lyubimova and Kovalevskaya 67] found a critical value of the Rayleigh number for the onset of buoyancy convection $R a_{c} \approx 84$.

Following the same approach already undertaken in our preceding analysis [68, here we report the nondimensional amplitude $A$ of the unsteady convective state for different values of $R a$ and the value of $R a_{c}$ obtained through (quadratic) extrapolations of $A$ to 0 (Table I). Even in this case it is straightforward to verify the good agreement between our calculations and the data available in the literature. Along the same lines, we wish to recall that in 68 , we found $R a_{c} \approx 472$ for $\vartheta=0.06$, the corresponding value determined by $\left[67\right.$ ] being $R a_{c} \approx 470$. For what concerns the validation of the Newtonian thermovibrational solver, the reader will find further details in 66.

\section{B. Mesh refinement study}

In the present work we consider a 3D geometry. Since conducting a grid independence-study would be extremely expensive from a computational point of view, following a common practice in the literature, here the mesh refinement study has been (initially) limited to the equivalent 2D configuration (assumed to have infinite extension along the third direction $z$ ). Such a modus operandi relies on the realization that since the considered 3D problem is isotropic with respect to the horizontal direction (i.e. no preferred direction exists in the $x z$ plane), the required grid resolution for the $x$ direction can also be applied to the perpendicular direction $z$.

As sensitive quantities for such investigation, we have analyzed the amplitude $\left(A_{u_{y}}\right)$ of the $y$ component of the 
TABLE I. Comparison with the linear stability analysis by Lyubimova and Kovalevskaya 67 for a layer of viscoelastic fluid delimited by top and bottom stress-free walls with $\operatorname{Pr}_{g}=$ $7, \Omega=26.5, \Gamma=1, \xi=0.1, \vartheta=0.38(\Sigma=1.6)$. The present results have been obtained using a structured mesh (2D simulation) with 14350 nodes and a domain having nondimensional horizontal extension 15 with periodic boundary conditions at the lateral boundaries. $A$ is the non-dimensional amplitude of the (axial) velocity signal in the centre of the layer.

\begin{tabular}{lc}
\hline \hline$R a$ & $A$ \\
\hline 100 & 4.60 \\
110 & 6.45 \\
120 & 7.98 \\
130 & 9.28 \\
79.5 (extrapolated) & 0 \\
84 (LSA) & 0 \\
\hline \hline
\end{tabular}

TABLE II. Mesh refinement study. Case $\operatorname{Pr}_{g}=7, \Omega=26.5$, $R a_{\omega}=1170, \vartheta=0.38, \Sigma=1.6$, viscoelastic fluid.

\begin{tabular}{ccc}
\hline \hline Mesh & $A_{u_{y}}$ & $\overline{N u}$ \\
\hline $150 \times 30$ & 24.46 & 1.13 \\
$150 \times 35$ & 24.73 & 1.14 \\
$200 \times 30$ & 26.67 & 1.15 \\
$200 \times 35$ & 27.15 & 1.15 \\
$300 \times 35$ & 29.52 & 1.18 \\
$410 \times 35$ & 31.23 & 1.21 \\
$450 \times 40$ & 31.87 & 1.22 \\
\hline \hline
\end{tabular}

u signal measured with a virtual probe located in the centre of the layer, and the time averaged value of the Nusselt number (defined by eq. 19p) for the following set of representative parameters: viscoelastic fluid with $P r_{g}=7, \Omega=26.5, R a_{\omega}=1170, \vartheta=0.38$ and $\Sigma=1.6$. As quantitatively substantiated by the data reported in Table II] a mesh with $300 \times 35$ cells can provide a reasonable level of grid independence. Indeed, when the number of points in the horizontal direction exceeds 300, the solution becomes essentially independent from the mesh (the

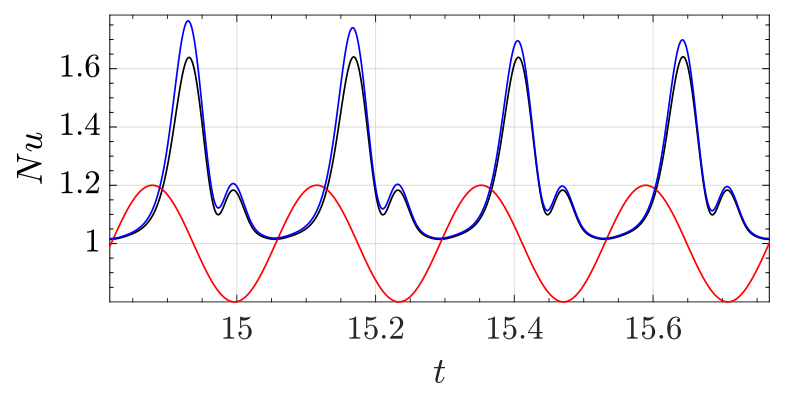

FIG. 2. Time evolution of the Nusselt number for 2 different densities of the mesh, in black (-) $200 \times 35 \times 200$, in blue (-) $300 \times 35 \times 300$, in red (-) the qualitative evolution of the acceleration. $3 \mathrm{D}$ simulations.

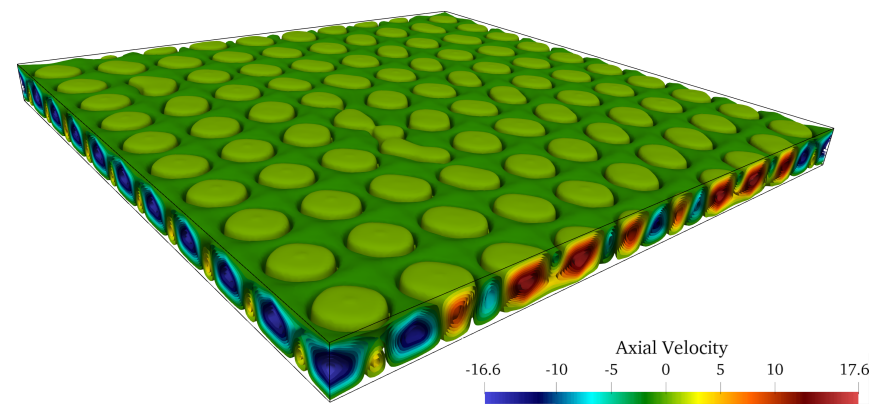

(a)

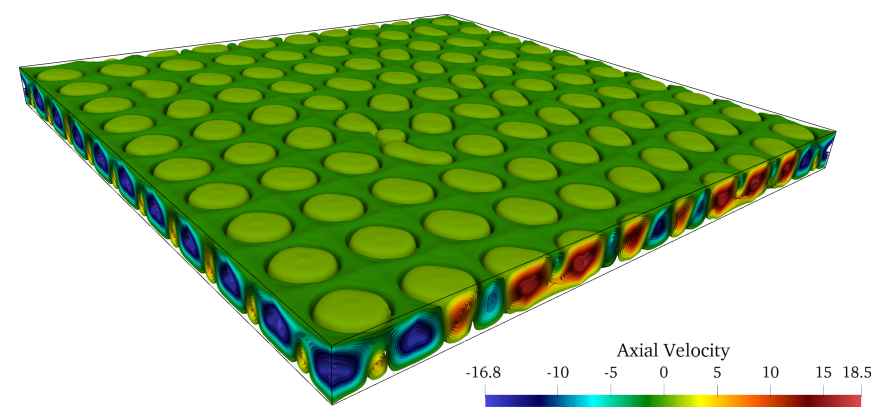

(b)

FIG. 3. 3D isosurfaces of the axial velocity for the case with $\operatorname{Pr}_{g}=7, \Omega=26.5, R a_{\omega}=1170, \xi=0.1, \vartheta=0.38, t=t_{0}=$ 14.92, (a) mesh $200 \times 35 \times 200$, (b) mesh $300 \times 35 \times 300$.

percentage variations for a variation of 100 points being $5 \%$ only for the instantaneous velocity and less than $3 \%$ for the average Nusselt number, a reasonable approximation from an engineering standpoint).

In order to verify the applicability of these findings to the complete (original) problem, the study has also been repeated considering a $3 \mathrm{D}$ parallelepipedic shallow domain having a square symmetry (yet referring to both the velocity signal and the Nusselt number). As witnessed by Figure 2, apart from a small vertical shift in the position of the curves, a change in the mesh has no impact on the system temporal response. The same conclusion also stems from Figure 3 where the 3D pattern (system behavior in space) is shown for the same conditions of Figure 2.

Taking into account the main implications of FIG. 2 and 3 in the present work most of simulations have been conducted using a grid with $200 \times 35 \times 200$ cells (a reasonable compromise between accuracy and the required simulation time).

\section{RESULTS}

The present study may be regarded in a certain way as an extension of the earlier work by Lyubimova and Kovalevskaya [67]. While in that analysis, emphasis was given to the influence exerted by vibrations on RayleighBénard convection for a comparable magnitude of the involved accelerations (steady gravity and $b \omega^{2}$ ), here we 
TABLE III. Extrapolation of the critical vibrational Rayleigh number $R a_{\omega, \mathrm{cr}}$ for a layer of viscoelastic fluid delimited by differentially heated solid-solid walls with $\operatorname{Pr}_{g}=7, \Omega=26.5$, $\xi=0.1$. The present results have been obtained using $2 \mathrm{D}$ simulation and a domain having $A R=15$ with cyclic boundary conditions at the lateral boundaries.

\begin{tabular}{cc}
\hline \hline$\vartheta$ & $R a_{\omega, \mathrm{cr}}$ \\
\hline Newtonian & 16070 \\
0.06 & 3245 \\
0.24 & 1207 \\
0.38 & 956 \\
\hline \hline
\end{tabular}

focus on pure thermovibrational flow. Moreover, we continue the line of inquiry originating from that work by probing the role of solid-solid boundary conditions and a different shape of the forcing "wave" (sinusoidal vibrations being considered in place of the "square" wave).

As assessing 3D effects through comparison with numerical simulations conducted under the constraint of two-dimensionality is always beneficial (actually the history of thermal convection is full of examples where focused comparisons of such a kind were instrumental in clarifying the nature of the dominant disturbances and possibles regimes of motion), we initially discuss some simulations carried out neglecting the $z$ direction (see section IVA the third dimension is re-introduced later in section IVB, which is entirely dedicated to the presentation and critical analysis of the related results).

On the one hand, this modus operandi is intentionally implemented to discern the ability of the flow to develop $3 \mathrm{D}$ components of velocity when the disturbances saturate their amplitude, on the other hand it fits into a more "practical" strategy where 2D simulations are used to determine the critical threshold for the onset of fluid motion (known to be driven by two-dimensional disturbances) with notable computational savings.

In particular, we consider a layer of an Oldroyd-B liquid having $\operatorname{Pr}_{g}=7$ and $\xi=0.1$ subjected to external vibrations with a frequency $\Omega=26.5$ (low frequencies regime). Three different level of elasticity are examined, i.e. $\vartheta=0.06,0.24$ and 0.38 corresponding to a value of $\Sigma=0.25,1.01$ and 1.6 respectively.

\section{A. 2D results and critical convective threshold}

This section is dedicated to the aforementioned $2 \mathrm{D}$ study, by which the value of the critical vibrational Rayleigh number $R a_{\omega, \text { cr }}$ is computed for any considered value of the elasticity number $\vartheta$. In particular, in order to mimic the typical approach envisaged by LSA (as already done in section IIIA to support the validation of the present solver), the needed threshold values are determined by extrapolating the amplitude $A$ of probe signals to 0 . For this purpose, a grid with $410 \times 35$ nodes is used. The ensuing results are presented in Table III
As quantitatively substantiated by this table, the critical threshold for the onset of convection strongly depends on the level of elasticity $(\vartheta)$. As expected, and in accordance with other studies on Rayleigh-Bénard (RB), Marangoni-Bénard (MB) and Marangoni or thermocapillary (TC) convection in viscoelastic liquids [30, 35, 36, 70, 82, 83, the bifurcation occurs at lower values of the governing parameter (in this case $R a_{\omega, \mathrm{cr}}$ ) for more elastic fluids. Interestingly, there is a shift of one order of magnitude if the results for Newtonian and viscoelastic fluids are compared (i.e. from $O\left(10^{4}\right)$ to $O\left(10^{3}\right)$ ).

In order to compare our results for different values of $\vartheta$ (the simulations with Newtonian fluid may be seen as the limit of $\vartheta \rightarrow 0$ ), we define a new parameter $r=R a_{\omega} / R a_{\omega, \mathrm{cr}}$, i.e. the ratio between the effective $R a_{\omega}$ used for the numerical simulation and the corresponding critical value (the one determined through extrapolation, reported in Table III). To fix the ideas, throughout the present study, this ratio is set to $r \approx 1.2$ (in other words, all the presented results exceed the critical threshold by a similar percentage).

Specific characterization of all these states is provided through the introduction of suitable quantities used to assess the "response" of the system from both fluiddynamic and thermal points of view. More precisely, we consider an axial velocity signal (the probe being located in the centre of the cavity) and the time evolution of the Nusselt number $N u(t)$ defined as:

$$
N u(t)=\frac{1}{A} \int_{A} \nabla T(t) \cdot \mathbf{n}_{\text {plate }} d A
$$

where $\mathbf{n}_{\text {plate }}$ in the unit vector normal to the boundary and $A$ is the area of the hot (or cold) plate (of course, in case of $2 \mathrm{D}$ simulations the area $A$ simply reduces to the length of the plate).

In addition, we introduce the time average Nusselt number $\overline{N u}$ by averaging the value of $N u(t)$ over its period of oscillation $T_{N u}$ :

$$
\overline{N u}=\frac{1}{T_{N u}} \int_{T_{N u}} N u(t) d t
$$

Following a logical approach, we conveniently start from the analysis of the velocity signals. Moreover, the Newtonian case is considered first (expected to be simpler in comparison to the cases where overstability enters the dynamics).

This is shown in FIG. 4a where the typical behavior with the fluid oscillating continuously between a quiescent state and convective motion can be recognized. This is in agreement with known solutions for square cavities (see e.g. 65, 66]). These peculiar dynamics are due to the continuous transition from stabilising (heating from above) to destabilising (heating from below) effects as the external dynamic force (resulting from the application of vibrations) changes its sign. As witnessed by the velocity signal spectrum (FIG. 5a), this periodic alternation has the same frequency of the external vibrations $\Omega$. Therefore, the state shown in this figure can be categorized as 


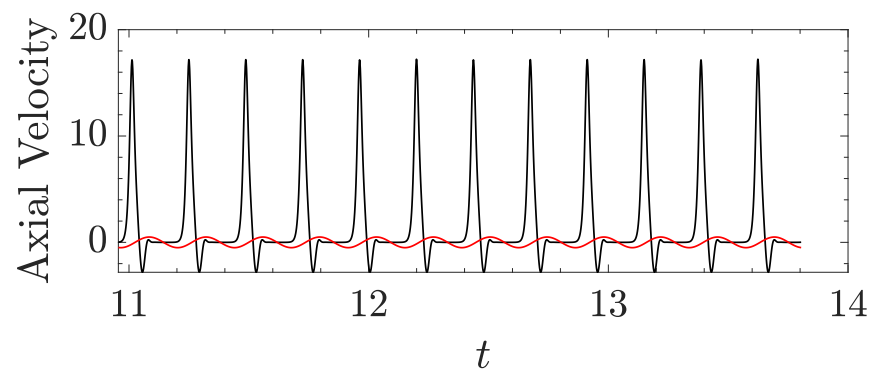

(a)

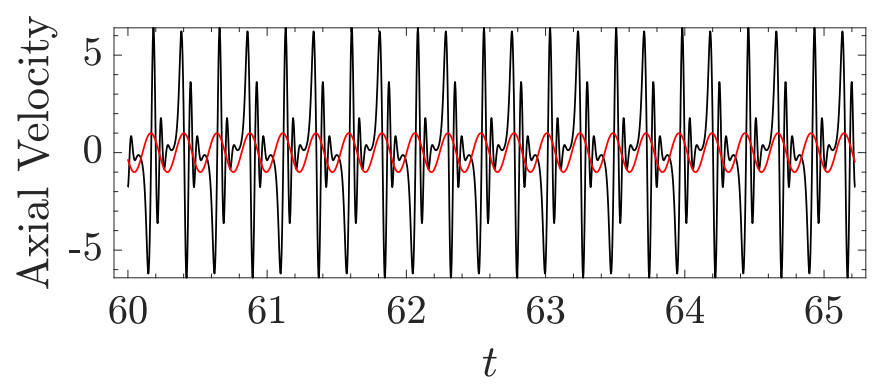

(b)

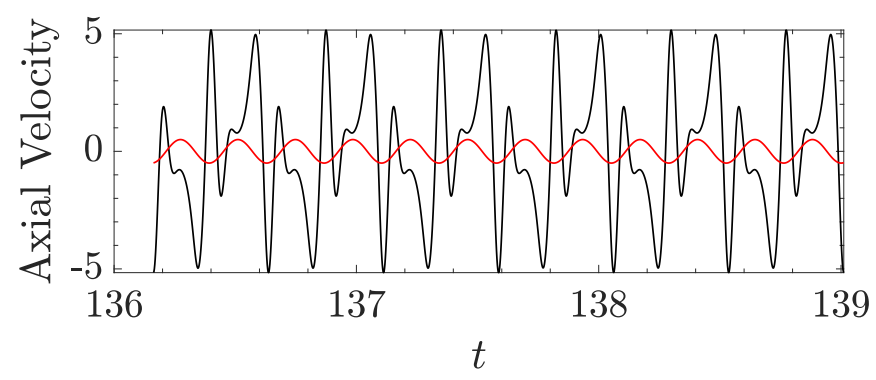

(c)

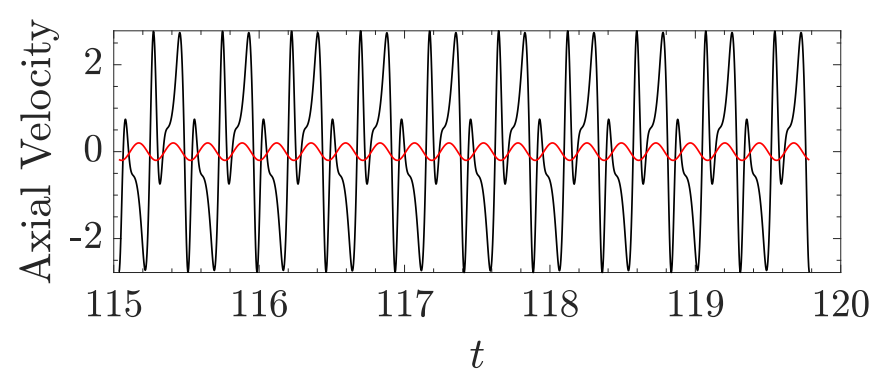

(d)

FIG. 4. Time evolution of the Axial velocity signal. The probe is located in the centre of the layer. Numerical simulation with $A R=15, \operatorname{Pr}_{g}=7, \Omega=26.5, \xi=0.1$ (a) Newtonian fluid, $R a_{\omega}=19750$, (b) $\vartheta=0.06, R a_{\omega}=3500$, (c) $\vartheta=0.24$, $R a_{\omega}=1475$, (d) $\vartheta=0.38, R a_{\omega}=1170$. In black (-) is represented the signal, in red (-) the evolution of $\mathbf{a}_{\omega}$.

a Synchronous-Periodic (SP) one ([65, 66]). Further confirmation for this interpretation stems from the evolution in time of the Nusselt number $N u(t)$ (FIG. 6a). When the fluid is in quiescent conditions, thermally diffusive conditions are established, and, accordingly, $N u(t)=1$.

The situation dramatically changes when the viscoelastic conditions are considered (see FIG.s $4 \mathrm{~b}, 4 \mathrm{c}$ and $4 \mathrm{~d}$.

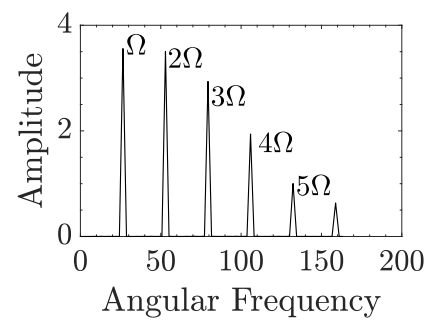

(a)

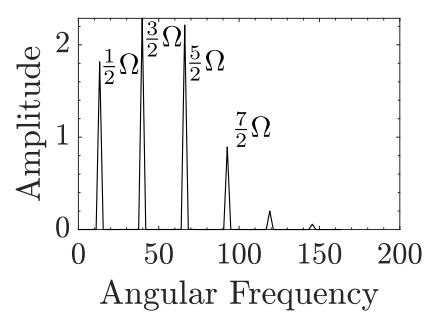

(c)

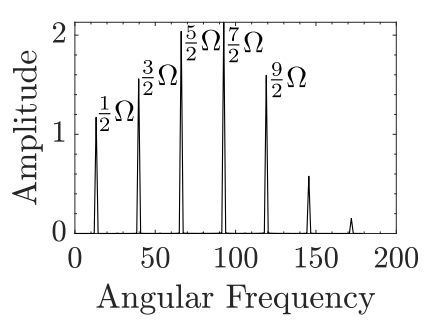

(b)

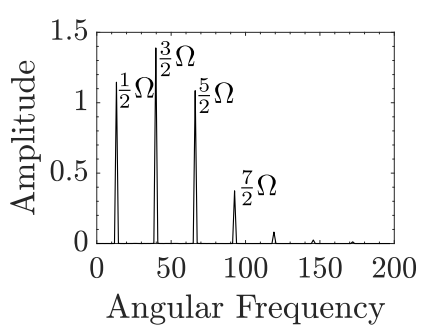

(d)
FIG. 5. Angular frequency of the Axial velocity signal. The probe is located in the centre of the layer. Numerical simulation with $A R=15, \operatorname{Pr}_{g}=7, \Omega=26.5, \xi=0.1$ (a) Newtonian fluid, $R a_{\omega}=19750$, (b) $\vartheta=0.06, R a_{\omega}=3500$, (c) $\vartheta=0.24$, $R a_{\omega}=1475$, (d) $\vartheta=0.38, R a_{\omega}=1170$.

As the reader will easily realize by inspecting these figures, no time interval exists where quiescent conditions are attained (Figure 4 and 6). Moreover, as one would expect in the light of the arguments provided before, for $\vartheta=0.24$ and $0.38, N u(t)$ is always higher than one.

Another notable modification in the dynamics concerns the frequency spectrum. The simple correspondence between the forcing frequency and the frequency of the induced velocity field is no longer a feature of these solutions. As evident in Figure 5 the flow displays frequencies that are smaller than the frequency of the imposed force (e.g., $\Omega / 2$ ). The behavior can therefore be classified as half-subharmonic (SU) ([66] and references therein).

Interestingly, even if the velocity spectrum is different for the two types of fluid, the Nusselt number spectrum, for the sake of brevity not reported, indicates that $N u(t)$ exhibits a SP evolution in all the cases analyzed (which indicates that the $\mathrm{SU}$ components of the temperature gradient at the wall reciprocally compensate when they are integrated).

The time average Nusselt Number $\overline{N u}$ also provides meaningful information. For the Newtonian fluid, $\overline{N u}=$ 1.16; it however increases to $\overline{N u}=1.22$ for $\vartheta=0.06$ and $\overline{N u}=1.24$ for $\vartheta=0.24$. Further increasing $\vartheta$ has the effect of making $\overline{N u}$ smaller $\left(\left.\overline{N u}\right|_{\vartheta=0.38}=1.21\right)$.

It can therefore be concluded that, for a viscoelastic fluid, the heat exchange due to convective phenomena is more effective $\left(\overline{N u}_{\text {Newt }}<\overline{N u}_{\text {visc }}\right)$. An explanation/justification for this trend can be elaborated in its simplest form on the basis of the argument that when 


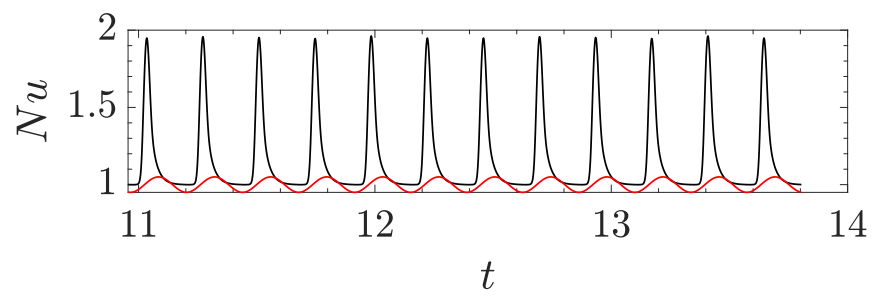

(a)

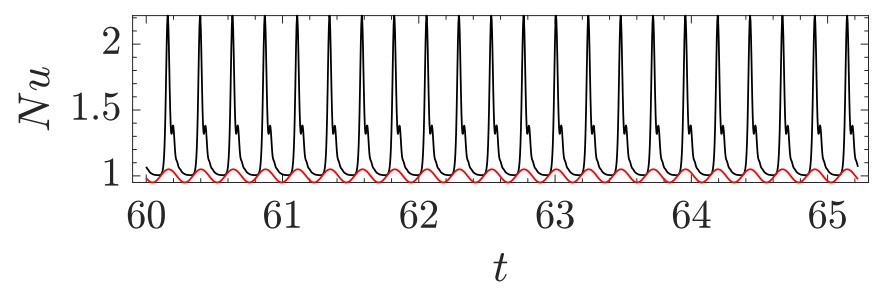

(b)

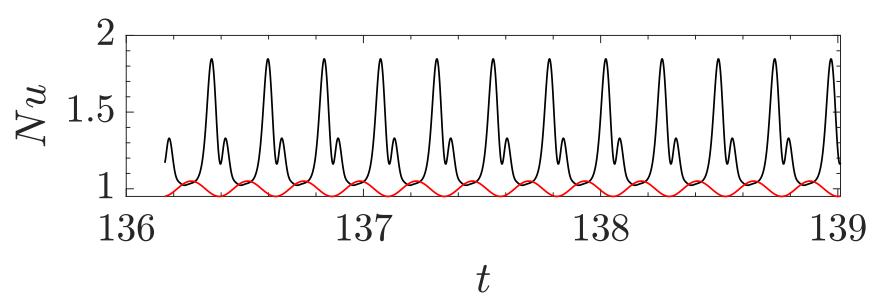

(c)

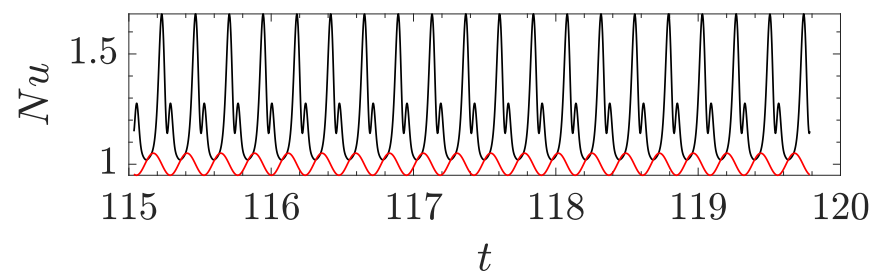

(d)

FIG. 6. Time evolution of $N u(t)$.Numerical simulation with $A R=15, P r_{g}=7, \Omega=26.5, \xi=0.1$ (a) Newtonian fluid, $R a_{\omega}=19750, \overline{N u}=1.16$, (b) $\vartheta=0.06, R a_{\omega}=3500, \overline{N u}=$ 1.22 , (c) $\vartheta=0.24, R a_{\omega}=1475, \overline{N u}=1.24$, (d) $\vartheta=0.38$, $R a_{\omega}=1170, \overline{N u}=1.21$. In black $(-)$ is represented $N u(t)$, in red (-) the evolution of $\mathbf{a}_{\omega}$.

overstability takes place, there are no transitional stages where the fluid is quiescent or thermal diffusive conditions are established.

As viscoelastic fluids are characterised by intrinsic 3D patterning behavior when the disturbances saturate their amplitude (see e.g. [27]), the next section is entirely dedicated to the presentation of the related 3D non-linear simulations.

\section{B. 3D pattering behavior and evolution}

A simple rationale for the expected 3D nature of the emerging flow when the constraint of two-dimensionality is removed can be gathered from the companion problem related to the Rayleigh-Bénard convection in a viscoelastic fluid. While in the Newtonian case, it is well known that the dominant flow structure in an infinite layer (or a sufficiently shallow enclosure) is simply given by a set of perfectly parallel rolls, convection in viscoelastic fluids is generally $3 \mathrm{D}$ even if relatively small values of the Rayleigh number are considered. As an example, Lappa and Boaro 27] have shown that the patterning behavior consists of parallel convective rolls that continuously break and re-assemble in a direction orthogonal to the initial one (this mechanism being periodic in time).

Following the same approach undertaken in section IVA however, first we consider the limiting condition of $\vartheta \rightarrow 0$, that is the Newtonian liquid. In this regard, we wish also to remark that in the present study all the 3D simulations (for both Newtonian and OldroydB fluids) have been conveniently initialised by remapping the $2 \mathrm{D}$ solutions in the equivalent $3 \mathrm{D}$ geometry. Obviously, this approach relies on the crucial information provided by earlier LSA studies, i.e. that the disturbances are $2 \mathrm{D}$ at the onset of convection and then they progressively become $3 \mathrm{D}$ as they saturate their amplitude (a concept which applies to buoyancy flow in both Newtonian and viscoelastic fluids [67]).

The first figure of the sequence related to the $3 \mathrm{D}$ results (FIG. 7) shows the time-evolution of the Newtonian fluid over half of the period of oscillation $T_{\Omega} / 2$ (the second half of the period is not represented as it simply corresponds to a motionless state).

A kind of pulsation occurs in the thermal and velocity fields. In particular, it can be seen that the initial diffusive state (FIG. 7a) is destabilised by the vibrations, which promote the onset of convection. Quasilongitudinal rolls arise and grow until they reach a cer-

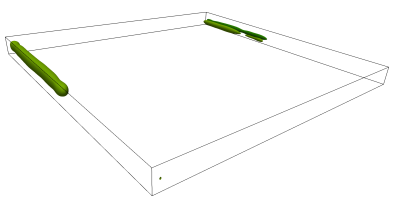

(a)

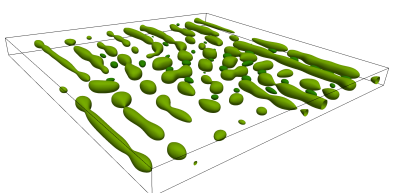

(c)

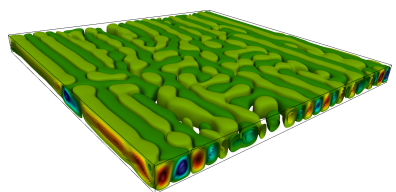

(b)

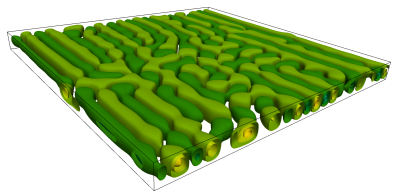

(d)

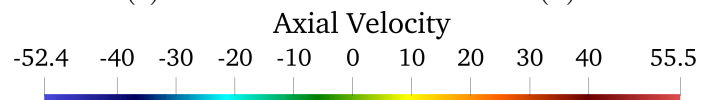

FIG. 7. Thermovibrational convection in a layer of Newtonian fluid delimited by differentially heated solid walls (snapshots of the isosurfaces of the y component of velocity evenly distributed over the first half period $\left.T_{\Omega}\right) . \operatorname{Pr}=7, \Omega=26.5$, $R a_{\omega}=19750$. 


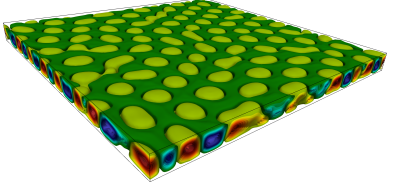

(a)

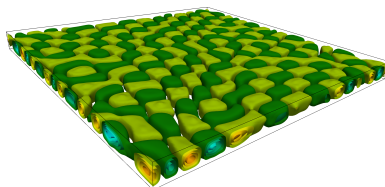

(e)

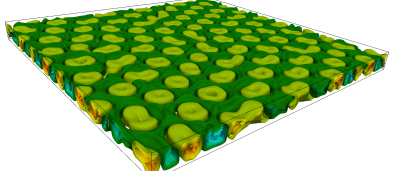

(b)

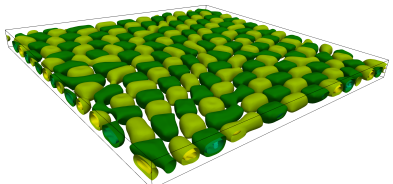

(f)

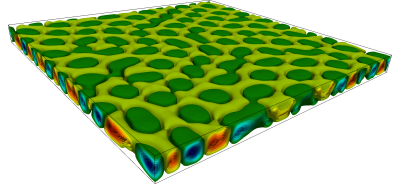

(c)

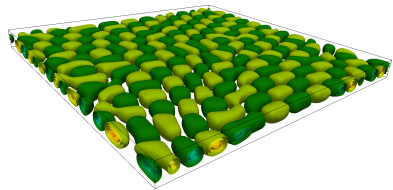

(g)

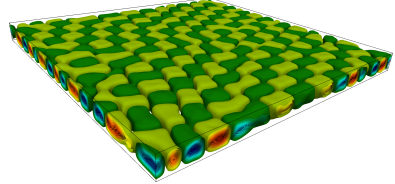

(d)

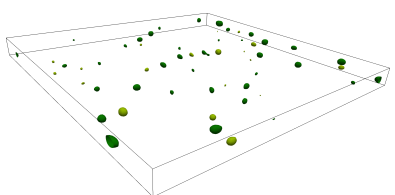

(h)

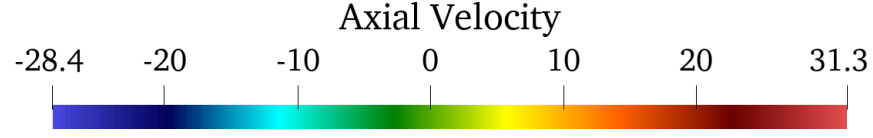

FIG. 8. Thermovibrational convection in a layer of Oldroyd-B fluid delimited by differentially heated solid walls (snapshots of the isosurfaces of the vertical component of velocity evenly distributed over the interval $\left.\mathcal{I}_{1}\right) . P r_{g}=7, \Omega=26.5, R a_{\omega}=3500$, $\xi=0.1, \vartheta=0.06$.

tain (maximum) dimension and intensity (FIG. 7b). At this stage, a pulsating behavior is enabled, as witnessed by FIG. 7c, where a decrease in the strength of convection can be noticed. The rolls then re-emerge with the same arrangement that they were displaying in FIG. 7b but with a different sense of rotation (FIG. 7d). However, in this transitional state, the velocity field does not reach the same intensity seen in the first part or the pulsation. This is due to the stabilizing effect of the vibrations, which in the meantime have changed direction, thereby tending to promote a new diffusive (quiescent) state.

This behavior has a one-to-one correspondence with the velocity signal. Put simply, both for the 2D (see FIG. 4a) and the 3D case (not reported here due to the similarity with the $2 \mathrm{D}$ data) a negative spike can be detected in the velocity signal. It corresponds to the aforementioned pulsation mechanism supported by a switch in the role of the dominant acceleration (from destabilizing to stabilizing).

As expected, this simple pulsating mechanism, which in the literature is also referred to as standing wave, becomes more involved when the Newtonian fluid is replaced by the equivalent viscoelastic liquid (same $P r_{g}$ and $r$, see Figures 8 14.

Given the complexity of the overall scenario, recalling briefly the trends already identified in the framework of the propaedeutical 2D analysis (section IV A is also beneficial.

As explained in that section, the convective phenomena that arise when $\vartheta$ is not zero can display a SU response to the harmonic action of the buoyancy force, i.e. the angular frequency of the thermal and fluid-dynamic fields can be $\Omega / 2$.

To fully understand the physical implications of this type of behavior, for instance, one may consider the velocity field $\mathbf{u}(t)$. By denoting with $t_{0}$ the time at which flow sampling is started, and recalling that $T_{\Omega}=2 \pi / \Omega$ is the period of the external vibrations, a $\mathrm{SU}$ behavior may be considered equivalent to stating that $\mathbf{u}\left(t_{0}\right)=$ $\mathbf{u}\left(t_{0}+2 T_{\Omega}\right)$. In order to fully characterize or describe the system response in this case, therefore, it is convenient to split ideally the oscillation period of the flow in four identical sub-intervals. In the following we will refer to these as $\mathcal{I}_{1}=\left[t_{0}, T_{\Omega} / 2\right], \mathcal{I}_{2}=\left[T_{\Omega} / 2, T_{\Omega}\right], \mathcal{I}_{3}=\left[T_{\Omega}, 3 / 2 T_{\Omega}\right]$ and $\mathcal{I}_{4}=\left[3 / 2 T_{\Omega}, 2 T_{\Omega}\right]$.

Along these lines, Figure 8 illustrates the evolution of the axial velocity (isosurfaces) in $\mathcal{I}_{1}$ for $\vartheta=0.06$ in the $3 \mathrm{D}$ case. It can be immediately seen that, interestingly, parallel rolls are no longer an emerging property of the flow. The parallel-roll-based structure is indeed taken over (FIG. 8a by a different (lattice-like) organization. While cold fluid moves from the cold plate towards the hot one, it is interrupted by localized islands of rising (hot) fluid. The isosurfaces of positive and negative axial velocity form two lattices of cold and hot fluid, perfectly entangled with one another.

As time passes, the aforementioned islands tend to split (FIG. 8b). A careful analysis of this mechanism (for the sake of brevity not illustrated in this study), has revealed that this phenomenon is caused by the nucleation of cold plumes in proximity to the cold plate. The currents of hot fluid eventually merge together and form a lattice mirror symmetric with respect to the one described at the beginning of the evolution (FIG. 8c).

In this regard, an analogy might be established with the earlier findings by Boaro and Lappa 68. Although obtained under the constraint of two-dimensionality (FENE-CR fluids evolving in a vibrated square cavity), those results revealed the nucleation of plumes or eddies close to the solid boundaries, which therefore should not be regarded as an exclusive prerogative of the dynamics reported here. 


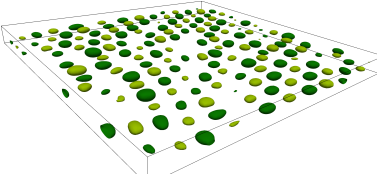

(a)

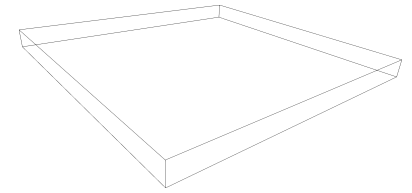

(c)

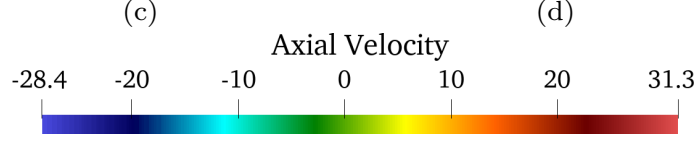

FIG. 9. Thermovibrational convection in a layer of Oldroyd-B fluid delimited by differentially heated solid walls (snapshots of the isosurfaces of the vertical component of velocity evenly distributed over the interval $\left.\mathcal{I}_{2}\right) . P r_{g}=7, \Omega=26.5, R a_{\omega}=$ $3500, \xi=0.1, \vartheta=0.06$.

This pulsating-like behavior is retained throughout $\mathcal{I}_{1}$; however, as the aforementioned stabilizing phase is approached, the intensity of the velocity field reduces progressively, until, at the end of the interval (FIG. 8h) an almost diffusive state is attained. Another key observation concerns the lattice structure seen in FIG. 8c, which with time is taken over by a checkerboard-like pattern.

Figure 9 refers to the next sub-interval $\left(\mathcal{I}_{2}\right)$. This figure is useful as it reveals that the flow field keeps pulsating (FIG.s 9a and 9b), until, at the end of this second phase, the intensity of the velocity field starts to increase again (FIG. 9d). In this part of the time-advancement it is evident that the layer displays a diffusive behavior in a subset of the interval (revealed by both the patterning behavior (FIG. 9c and the evolution of $N u(t)$, not reported here for the $3 \mathrm{D}$ case due to the similarity with the $2 \mathrm{D}$ data of Figure 6 .

The next figure of the sequence (Figure 10) illustrates what happens when the third sub-interval is entered. As a fleeting glimpse into this figure would confirm, the pattern evolution is similar in $\mathcal{I}_{1}$ and $\mathcal{I}_{3}$. Eventually, in the interval $\mathcal{I}_{4}$ the evolution repeats itself in a similar way to $\mathcal{I}_{2}$ and, for sake of brevity, we omit it here.

An increase in the elasticity parameter $(\vartheta=0.24)$ does not lead to substantial changes in the dynamics. By inspecting FIG. 11a, however, a new feature can be highlighted: the interconnection between the different islands of hot fluid tends to become more evident (they form a kind of "net"). Moreover, the morphology of the islands is slightly different (their inner boundary is no longer circular and resembles the shape of a "heart"). The evolutionary progress in time is still similar to that described before, e.g. in FIG. 11b the small rolls of cold fluid in proximity to the cold wall are visible. The intensity of the velocity still undergoes a decrease after some time, but less than in the previous case.

Eventually, in FIG. 11c the inversion of the flow axial direction takes place. At this stage, however, things become more complex. Indeed, the hot fluid plumes that are now detached one from each other, start merging in proximity to the cold boundary, pushing the cold fluid to the hot plate (and vice-versa) (see FIG. 11d and 11e). The hot fluid close to the cold wall is therefore cooled, the intensity of the velocity field becomes smaller and new cold plumes tend to be formed again.

Other two "inversions" take place between FIG.s 11e $11 \mathrm{~h}$ however, approaching the time interval $\mathcal{I}_{2}$ the intensity of $\mathbf{u}$ diminishes. It is also worth observing that now, at the end of $\mathcal{I}_{1}$ the flow-field still displays convective structures.

This means that, unlike the case with $\vartheta=0.06$, convection is not suppressed (Figure 12). In particular, in $\mathcal{I}_{2}$ small pulsations of the rolls are still present. However, this oscillatory mechanism is limited to a weak modulation of the intensity of the velocity field, which never changes its sign. Put differently, the modulation is not strong enough to cause the inversion of the velocity. At the end of $\mathcal{I}_{2}$ a situation similar to the one described in the previous case is attained $\left(\mathbf{u}\left(t_{0}, \mathbf{x}\right)=\mathbf{u}\left(t_{0}+T_{\Omega}, \mathbf{x}+\mathbf{a}\right)\right)$. Therefore, for the sake of brevity, the second part of the oscillation period is glossed over here (i.e. the evolution of the pattern in $\mathcal{I}_{3}$ and $\mathcal{I}_{4}$ for $\left.\vartheta=0.24\right)$.

As a final case, we analyze $\vartheta=0.38$ (Figure 13.

Surprisingly, the structure of the lattice is now perfectly ordered (the related convective features are evenly distributed in space, FIG. 13a. The increase in the elasticity parameter has the effect to give to cold plumes a well defined and rounded shape. This kind of self organised structure is reminiscent of the so called complex order structures originally reported by Rogers and coworkers 84 87 for modulated gravitational convection in Newtonian fluids. Even for this case the entanglement between cold and hot fluid lattices can be clearly observed.

The dynamics are similar to that analysed for lower values of $\vartheta$, i.e. the formation of colder fluid eddies (FIG. 13b the visible part is actually the imprint of the small eddies on the main structures) is followed by the weakening of the velocity field intensity that leads to an inversion of the plume configuration (FIG. 13d). In particular, we can observe smaller blobs of hot fluid moving toward the centre of the main plumes.

As evident in FIG. 13d, a checkerboard distribution is eventually established. Blobs of hot and cold fluid occupy the whole vertical space, i.e. there is not interconnection and stratification of the plumes (as it was, e.g., in FIG. 13a). Now the isolated plumes (see e.g. the ones representing the rising fluid) tend to merge together (FIG. 13e in such a way that all the blobs are interconnected and the stratification is established once more.

Later on, new cold eddies (not visible) start forming again. This mechanism leads to the fascinating convective structure depicted in FIG. 13f It is reminiscent of 


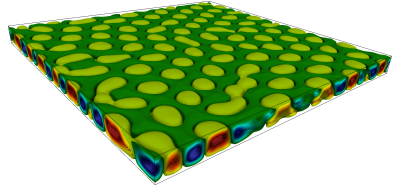

(a)

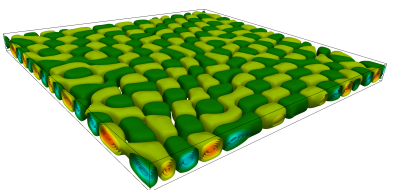

(e)

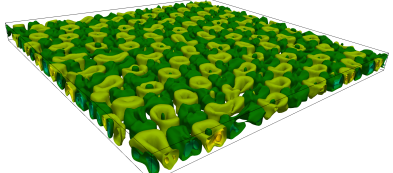

(b)

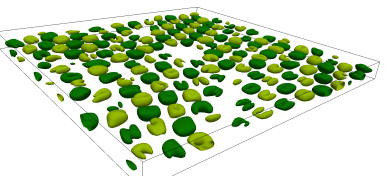

(f)

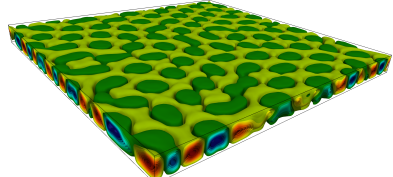

(c)

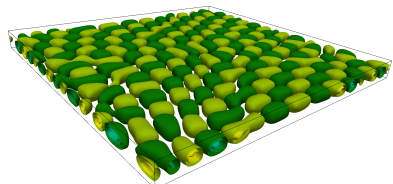

(g)

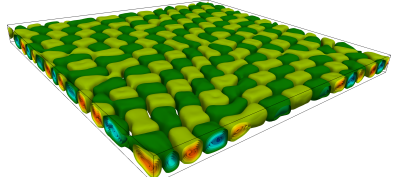

(d)

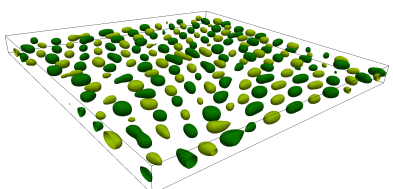

(h)

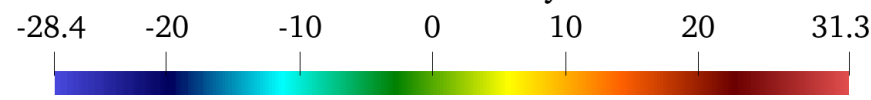

FIG. 10. Thermovibrational convection in a layer of Oldroyd-B fluid delimited by differentially heated solid walls (snapshots of the isosurfaces of the vertical component of velocity evenly distributed over the interval $\left.\mathcal{I}_{3}\right) . \operatorname{Pr}_{g}=7, \Omega=26.5, R a_{\omega}=3500$, $\xi=0.1, \vartheta=0.06$.

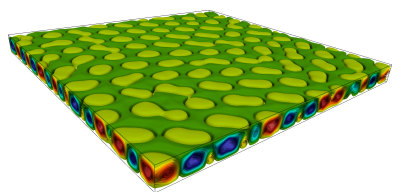

(a)

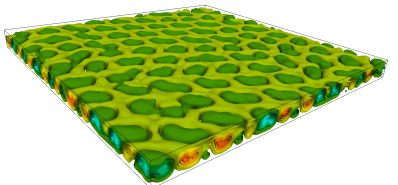

(e)

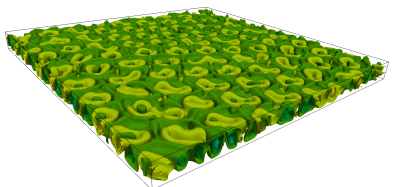

(b)

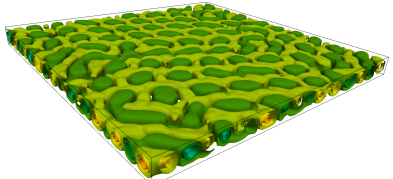

(f)

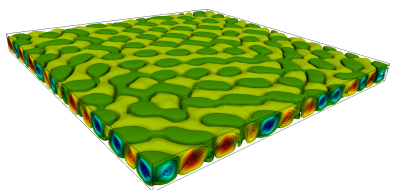

(c)

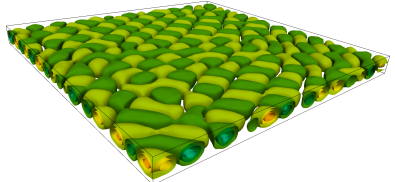

(g)

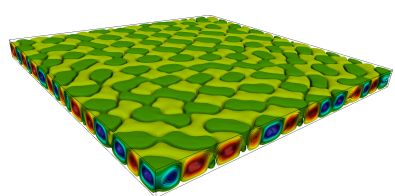

(d)

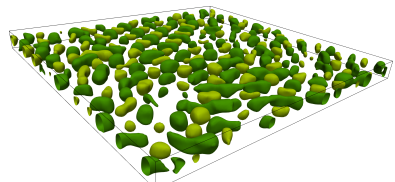

(h)

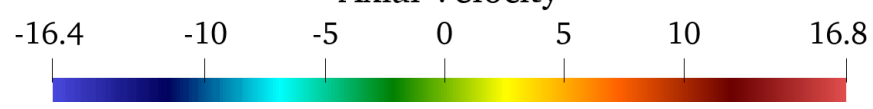

FIG. 11. Thermovibrational convection in a layer of Oldroyd-B fluid delimited by differentially heated solid walls (snapshots of the isosurfaces of the vertical component of velocity evenly distributed over the interval $\mathcal{I}_{1}$ ). $\operatorname{Pr}_{g}=7, \Omega=26.5, R a_{\omega}=1475$, $\xi=0.1, \vartheta=0.24$.

the so called bimodal convection observed in Newtonian fluids (see e.g. [1, 88]).

A direct comparison of FIG.s $8 \mathrm{~h}$ and $11 \mathrm{~h}$ and $13 \mathrm{~h}$ finally reveals that the higher the elasticity of the fluid, the larger intensity of velocity field at the end of $\mathcal{I}_{1}$.

For the sake of completeness, the pattern evolution in $t \in \mathcal{I}_{2}$ is represented in Figure 14. While, on the one hand, this figure shows that the dominant mechanism essentially resembles that already described for $\vartheta=0.24$, on the other hand, it qualitatively supports the realization that in these circumstances the rolls have accumulated enough energy to invert their sense of rotation.

\section{DISCUSSION}

As a relevant means to provide additional insights into the dynamics described in section IV FIG. 15 provides an alternate representation of the flow (for the different conditions considered in the present work) by revealing the evolution of the axial velocity (perpendicular to the plates) along a fixed line parallel to the $x$ direction (belonging to the mid-height plane, i.e. $0<x<15, y=0.5$ and $z=7.5$ ) as a function of time (over the interval $2 T_{\Omega}$ ).

This figure is instrumental in showing synthetically the various flow weakening and strengthening effects reported in section IV as a function of the elasticity parameter. Along these lines, examination of another quantity, 


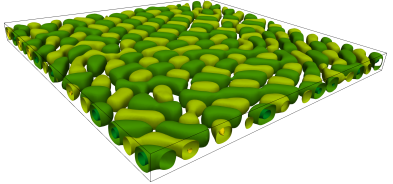

(a)

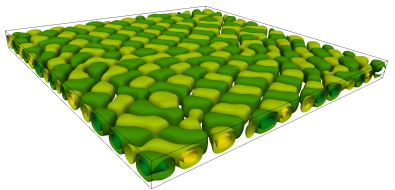

(c)

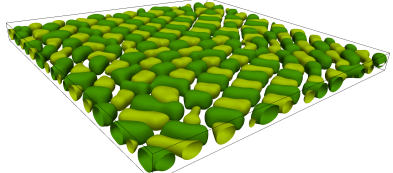

(b)

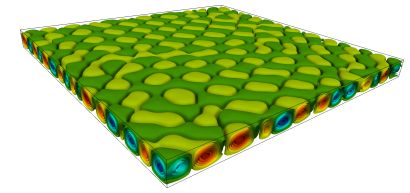

(d)

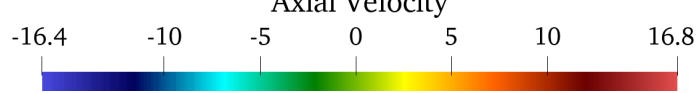

FIG. 12. Thermovibrational convection in a layer of OldroydB fluid delimited by differentially heated solid walls (snapshots of the isosurfaces of the vertical component of velocity evenly distributed over the interval $\left.\mathcal{I}_{2}\right) . \operatorname{Pr}_{g}=7, \Omega=26.5$, $R a_{\omega}=1475, \xi=0.1, \vartheta=0.24$.

i.e. $\operatorname{tr}(\tilde{\boldsymbol{\tau}})$, is even more useful. Indeed, this parameter is known for its ability to represent (be proportional to) the local elastic energy stored in the fluid (see e.g. [89]; owing to this relationship, in the following we will simply refer to it as "elastic energy"). These data (Figure 16) obviously serve as another source of information for the interpretation of the fascinating mechanisms described before.

In particular, the significance of this additional figure resides in its ability to make evident that the elastic energy accumulated by the flow in the transitional stages of evolution where the acceleration is destabilizing is released more gradually (when the acceleration changes sign) for larger values of $\vartheta$ (compare Figure 16a, b and c).

In this regard, it is also worth recalling the parameter $\Sigma$, i.e. the ratio between the relaxation time $\lambda$ and the period of external vibration $T_{\omega}^{*}$, formally defined in section II. In the present work, since the vibrations frequency is constant, $\Sigma$ is a linear function of $\vartheta$. In particular, its values are $\Sigma=0.25,1.01$ and 1.6 for $\vartheta=0.06$, 0.24 and 0.38 , respectively. One may therefore argue that when $\vartheta=0.06$ the polymer molecule has sufficient time to relax to the initial position before the cycle of external vibration is finished. Obviously, similar considerations can be used to interpret the other two cases for which the molecules relaxation time and the vibration cycle coincide (for $\vartheta=0.24$ ) or for which the polymer molecule cannot relax completely within the oscillation period (for $\vartheta=0.38$ ).

These facts can directly be connected to the ability of the polymeric liquid to retain and store elastic energy and, to sustain specific convective states along the oscillation period.

The next figure (Figure 17) provides the spatial dis- tribution of this energy at the two characteristic times $t=t_{0}$ and $t=t_{0}+T_{\Omega} / 2$, which paves the way to a brief excursus on the analogies or affinities between this kind of dynamics and the very similar ones previously identified by Rogers et al. 84 87. for modulated Rayleigh-Bénard convection in Newtonian fluids.

As spontaneous symmetry breaking and pattern formation are universal phenomena observed in a wide variety of non-equilibrium systems, here we use this realization as an opportunity to develop an analogy which may help the reader to glean hints and draw inferences about what factors may facilitate the formation of patterns like those reported in the present work.

Along these lines, it is worth recalling that canonical planforms such as those formed by Rayleigh-Bénard (RB) or Marangoni-Bénard (MB) convection display relatively simple basic structures, generally consisting of stripes (rolls) or hexagons. It is also known that, in some circumstances, such structures tend to be distorted on long scales, that is, the pattern might have disordered local orientations, and/or contains defects of various types such as domain walls, dislocations or disclinations. These geometrical features can make the flow slightly more complex than the perfect planforms expected for the idealized infinite layer case and/or for conditions very close to the critical ones. Another known (although rarer) kind of complexity is represented by "complex order", namely, a structure where order manifests itself in a nontrivial way. Exemplars pertaining to this class of patterns are "quasicrystalline states"; their distinguishing mark is the ability to develop a complicated spatial structure that never repeats itself, but is well ordered in the Fourier space.

Relevant theoretical background for this category of phenomena can be found in the earlier work by Pismen and Rubinstein [90] where the concept of complex order was introduced on a set of rigorous bases. Towards the end to interpret the present results, here we limit ourselves to discussing the most fundamental underlying idea, that is, the relationship between complex order and the initial isotropy of the considered system.

Put simply such a notion can be expressed as follows: transitions to non-symmetric states in isotropic systems generally imply a preferred wavelength but no preferred direction; as a natural consequence of this property, an indefinite number of modes can be excited in principle at the same time, with the related wave vectors having the same absolute value but being directed in arbitrary directions. In such a process, in some special conditions, crystalline structure can be developed due to nonlinear interactions among these modes as they grow and saturate their amplitude.

Additional insights into these concepts (these being beyond the scope of the present section) can be found in the study by Lifshitz 91] where these phenomena have been further split in quasi-periodic crystals and superlattices depending on certain properties of the involved modes and their spatial relationships.

Coming back to the original problem of interest in the 


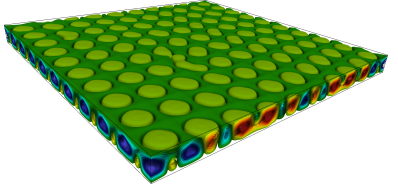

(a)

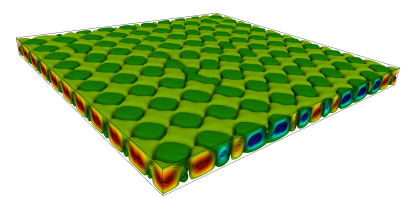

(e)

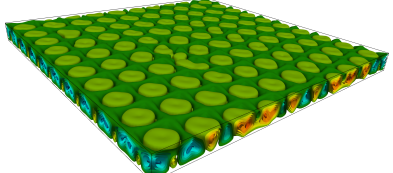

(b)

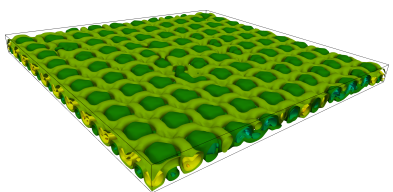

(f)

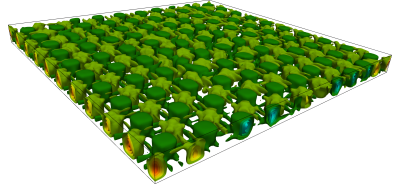

(c)

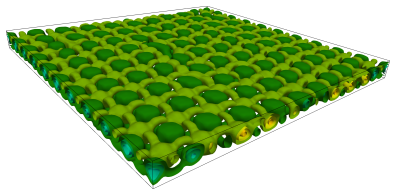

(g)

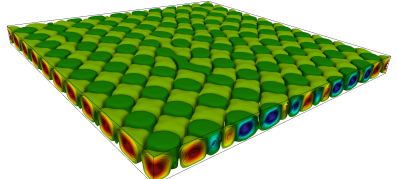

(d)

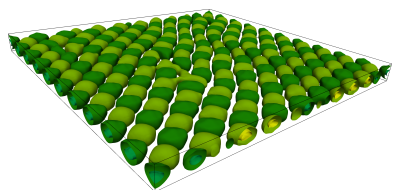

(h)

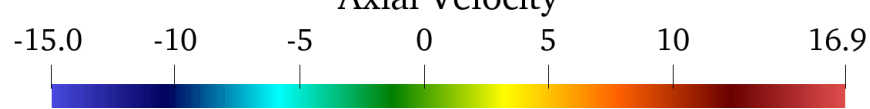

FIG. 13. Thermovibrational convection in a layer of Oldroyd-B fluid delimited by differentially heated solid walls (snapshots of the isosurfaces of the vertical component of velocity evenly distributed over the interval $\left.\mathcal{I}_{1}\right) . P r_{g}=7, \Omega=26.5, R a_{\omega}=1170$, $\xi=0.1, \vartheta=0.38$.

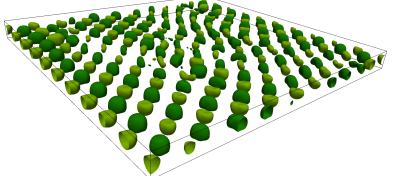

(a)

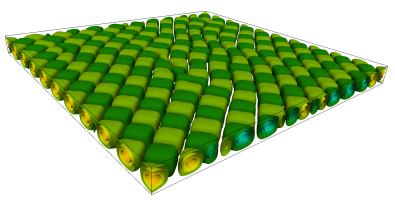

(c)

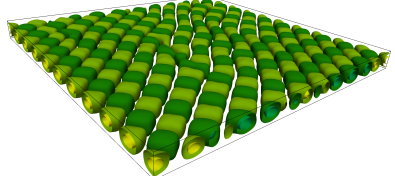

(b)

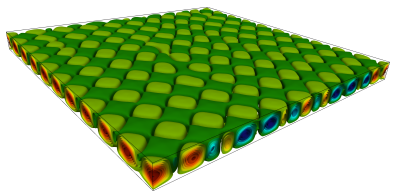

(d)

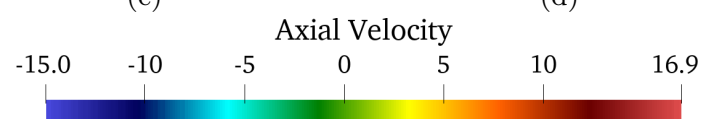

FIG. 14. Thermovibrational convection in a layer of OldroydB fluid delimited by differentially heated solid walls (snapshots of the isosurfaces of the vertical component of velocity evenly distributed over the interval $\left.\mathcal{I}_{2}\right) . \operatorname{Pr}_{g}=7, \Omega=26.5$, $R a_{\omega}=1170, \xi=0.1, \vartheta=0.38$.

present study, i.e. vertically-oscillated layer, it is worth re-emphasizing that such a system preserves the original isotropy of the classical RB and MB systems. The analogy with the systems investigated by Rogers and coworkers can be pursued further considering that, as illustrated in section IV the present layer has displayed the ability to develop both harmonic $(\mathrm{H})$ and subharmonic $(\mathrm{S})$ temporal responses when viscoelasticty enters the dynamics even though conditions very close to the critical ones are considered $(r=1.2)$.

Rogers and coworkers 84 87] had considered RB convection in a Newtonian fluid $(P r=0.93)$ under the effect of vibrations. In such conditions (and in analogy with the dynamics reported in section IV they found both $(\mathrm{H})$ and $(\mathrm{S})$ responses and even circumstances where both behaviors were present (in a sub-region of the space of parameters originating from a "bicritical" point). Over a parameter range where the mechanisms $(\mathrm{H})$ and $(\mathrm{S})$ have comparable influence, the spatial scales associated with both responses were found to coexist and lead to "resonant states" resulting in complex, highly ordered patterns.

These phenomena and related conditions may be considered formally similar to those examined in the present work where superlattice-like structures have been observed in combination with the existence of two distinct spatial scales, each displaying a different temporal dependence (the reader being referred again to the information reported in section IV]. In the framework of the analogy proposed here, one may argue that while in Newtonian fluids the existence of disturbances with different temporal scales does require concurrent mechanisms driving the flow (gravity and vibrations in the case of Rogers and coworkers), in the present problem these are naturally present as a results of the ability of viscoelasticity to cause the coexistence of harmonic and subharmonic modes with distinct critical wavenumbers. Put simply, the presence of a second driving force is not needed as viscoelasticity provides the fluid with natural capacity to develop disturbances with various wavelengths (this being through the ability of the molecules of dispersed polymer to stretch and deform under the effect of a primary flow and exert a back influence on the flow which generated the deformation, thereby leading to the emergence of a "secondary" flow); the reader specifically interested in such arguments, may also consider Boaro and Lappa 68 . 


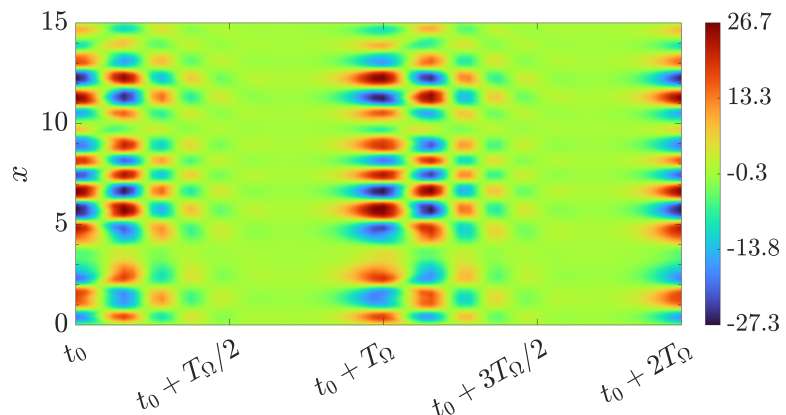

(a)

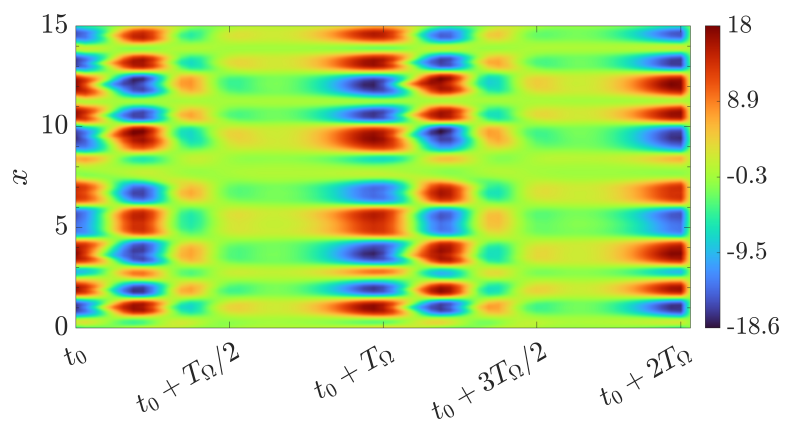

(b)

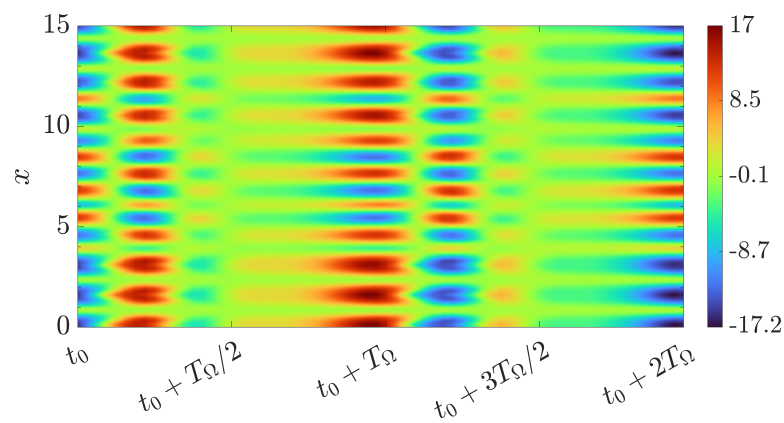

(c)

FIG. 15. Color field of axial velocity ( $y$ component of $\mathbf{u}$ ) as a function of the time and the $x$ coordinate in the centre of the cavity. $0<x<15, y=0.5, z=7.5$. (a) $\vartheta=0.06$, (b) $\vartheta=0.24$, (c) $\vartheta=0.38$.

\section{CONCLUSIONS}

Thermovibrational flow in a layer of viscoelastic fluid with imposed vibrations parallel to the temperature gradient has been investigated.

This study has opened a new path to a much better understanding of this attractive system, which has many interesting aspects. In particular, we have combined concepts from disparate and segregated research areas to provide better knowledge of viscoelastic thermovibrational convection in specific sub-regions of the space of parameters where a Newtonian fluid would display relatively simple (canonical) behaviors.

It has been proven that the onset of thermovibrational convection in Oldroyd-B fluids occurs for values of the control parameter $\left(R a_{\omega}\right)$, which are one order of magni-

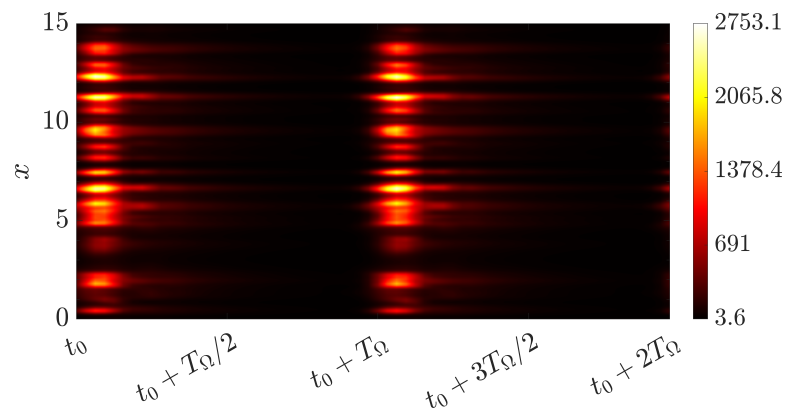

(a)

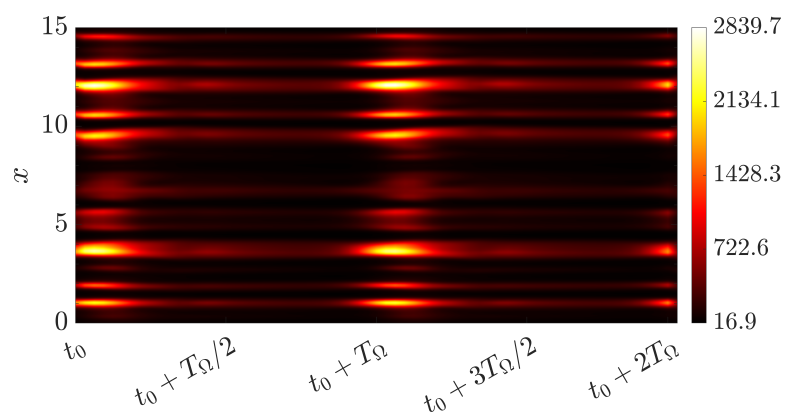

(b)

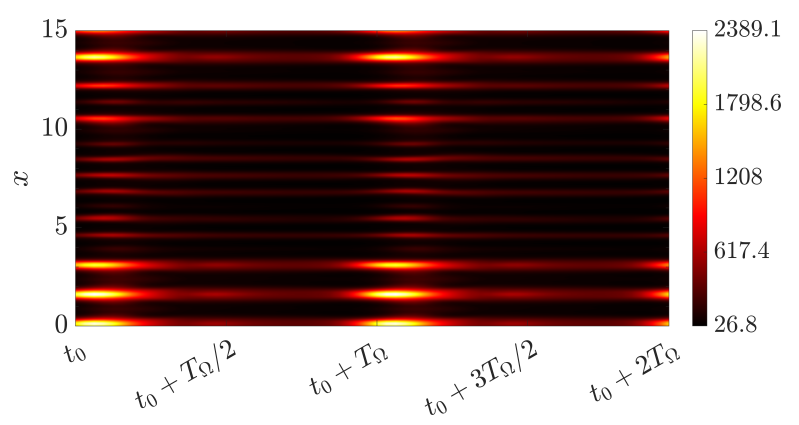

(c)

FIG. 16. Color field of $\operatorname{tr}(\tilde{\boldsymbol{\tau}})$ as a function of the time and the $x$ coordinate in the centre of the cavity. $0<x<15, y=0.5$, $z=7.5$. (a) $\vartheta=0.06$, (b) $\vartheta=0.24$, (c) $\vartheta=0.38$.

tude smaller than the equivalent threshold to be exceeded in the companion Newtonian case, thereby lending evidence to the applicability of the concept of "overstability" to these circumstances.

We have shown that the relatively obvious dynamics typical of slightly super-critical states in Newtonian fluids, where the flow is characterized by the existence of parallel rolls that periodically disappear and re-emerge with different sense of rotation are taken over for viscoelastic fluids by a kind of complex order driven by the interplay of the time-varying (stabilizing/destabilizing) role of the vibration induced acceleration and the ability of the fluid to store and release elastic energy.

The stored energy allows the viscoelastic medium to sustain a convective flow even in the stages where the external force plays a stabilizing role (i.e. it tends to suppress fluid motion). In this specific phases, the inten- 


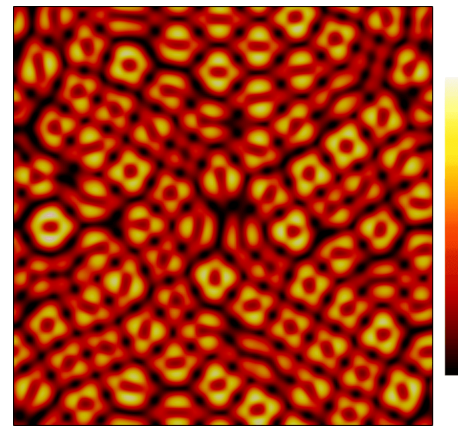

(a)

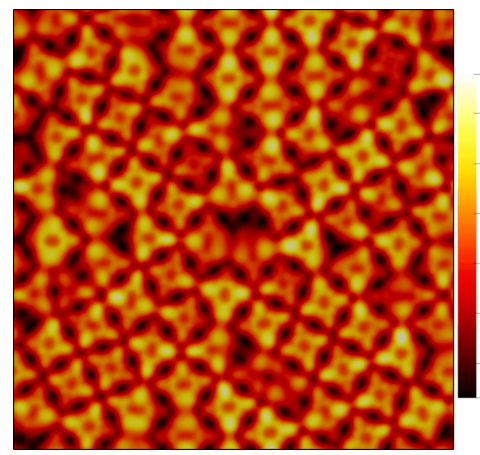

(d)

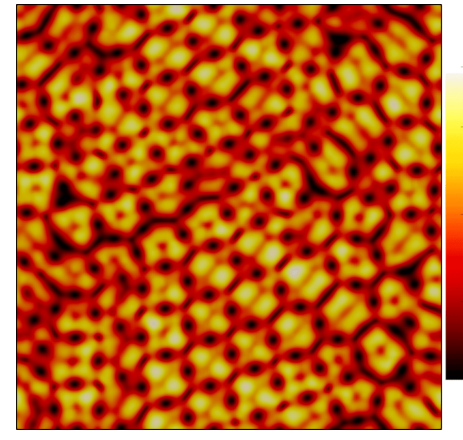

(b)

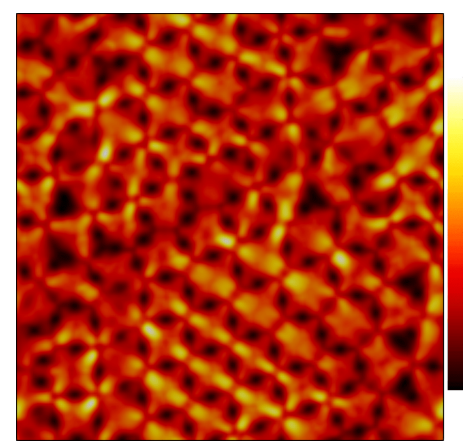

(e)

1839.2
1500
1000
500
52.5

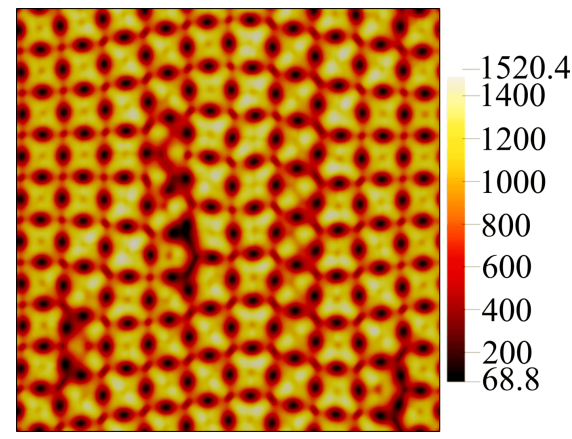

(c)

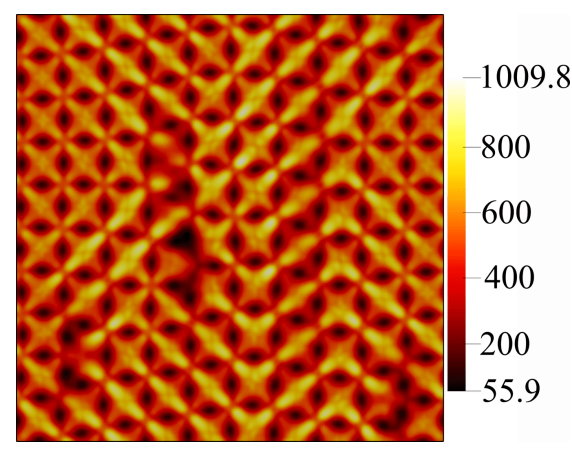

(f)

FIG. 17. Distribution of $\operatorname{tr}(\tilde{\boldsymbol{\tau}})$ on the $x z$ plane at $y=1$ (cold plate). (a)-(c) $\vartheta=0.06,0.24$ and 0.38 respectively, $t=t_{0}$. (d)-(f) $\vartheta=0.06,0.24$ and 0.38 respectively, $t=t_{0}+T_{\Omega} / 2$.

sity of the flow field is directly related to the quantity of energy that the fluid could store in the preceding stage. In turn, such a quantity is directly related to the elasticity number, i.e. the fluid elasticity level, and to the ratio between elastic and dynamic oscillation characteristic times (i.e. the parameter $\Sigma$ ).

In the attempt to interpret the peculiar nature of the observed 3D planforms and the overall related scenario we have emphasized on some prior investigations that can be linked to the general problem relating to the emergence of superlattices in isotropic systems. Building on these works, we have introduced an interesting analogy and some new observations, both general and systemspecific, which will require additional attention in the future.
[1] F. H. Busse, The sequence-of-bifurcations approach towards understanding turbulent fluid flow, Surveys in Geophysics 24, 269 (2003).

[2] A. Mialdun, I. I. Ryzhkov, D. E. Melnikov, and V. Shevtsova, Experimental evidence of thermal vibrational convection in a nonuniformly heated fluid in a reduced gravity environment, Phys. Rev. Lett. 101, 084501 (2008)

[3] V. Shevtsova, I. I. Ryzhkov, D. E. Melnikov, Y. A. Gaponenko, and A. Mialdun, Experimental and theoretical study of vibration-induced thermal convection in low gravity, Journal of Fluid Mechanics 648, 53-82 (2010)

[4] A. Vorobev and T. P. Lyubimova, Vibrational convection in a heterogeneous binary mixture. part 1. timeaveraged equations, Journal of Fluid Mechanics 870, 543-562 (2019)

[5] D. V. Lyubimov, T. P. Lyubimova, A. A. Cherepanov,
N. I. Lobov, V. Vasilyev, and B. Roux, Vibration effect on morphological instability of planar solidification front, Journal of Crystal Growth 303, 269 (2007), proceedings of the Fifth Workshop on Modeling in Crystal Growth.

[6] V. Shevtsova, T. P. Lyubimova, Z. Saghir, D. Melnikov, Y. Gaponenko, V. Sechenyh, J. C. Legros, and A. Mialdun, IVIDIL: on-board g-jitters and diffusion controlled phenomena, Journal of Physics: Conference Series 327, 012031 (2011)

[7] V. Shevtsova, A. Mialdun, D. Melnikov, I. Ryzhkov, Y. Gaponenko, Z. Saghir, T. Lyubimova, and J. C. Legros, The ividil experiment onboard the iss: Thermodiffusion in the presence of controlled vibrations, Comptes Rendus Mécanique 339, 310 (2011).

[8] A. Gelfgat, Instability of natural convection of air in a laterally heated cube with perfectly insulated horizontal boundaries and perfectly conducting spanwise bound- 
aries, Phys. Rev. Fluids 5, 093901 (2020).

[9] S. Kaddeche, D. Henry, and H. Benhadid, Magnetic stabilization of the buoyant convection between infinite horizontal walls with a horizontal temperature gradient, Journal of Fluid Mechanics 480, 185-216 (2003)

[10] Y. Yan, V. Shevtsova, and M. Z. Saghir, Numerical study of low frequency g-jitter effect on thermal diffusion, Fluid Dynamics \& Materials Processing 1, 315 (2005)

[11] M. Sohail and M. Z. Saghir, Three-dimensional modeling of the effects of misalignment on the growth of ge1-xsix by the traveling solvent method, Fluid Dynamics \& Materials Processing 2, 127 (2006)

[12] M. Lappa, Patterning behavior of gravitationally modulated supercritical marangoni flow in liquid layers, Phys. Rev. E 93, 053107 (2016)

[13] M. Renardy, High weissenberg number asymptotics and corner singularities in viscoelastic flows, in IUTAM Symposium on Non-linear Singularities in Deformation and Flow, edited by D. Durban and J. R. A. Pearson (Springer Netherlands, Dordrecht, 1999) pp. 13-20.

[14] A. Bonito, P. Clément, and M. Picasso, Viscoelastic flows with complex free surfaces: Numerical analysis and simulation, in Numerical Methods for Non-Newtonian Flu$i d s$, Handbook of Numerical Analysis, Vol. 16, edited by R. Glowinski and J. Xu (Elsevier, 2011) pp. 305 - 369.

[15] H. Demir, R. W. Willianms, and F. T. Akyıldız, The singularities near the corner of a viscoelastic fluid in a $2 \mathrm{~d}$ cavity, Mathematical and Computational Applications 4, 39 (1999)

[16] D. Siginer, Stability of Non-Linear Constitutive Formulations for Viscoelastic Fluids (SpringerBriefs in Applied Sciences and Technology, 2014).

[17] R. G. Owens and T. N. Phillips, Computational Rheology (Imperial College Press, 2002) https://www.worldscientific.com/doi/pdf/10.1142/p160

[18] M. Lappa, Thermally-driven flows in polymeric liquids, in Reference Module in Materials Science and Materials Engineering (Elsevier, 2020).

[19] T. Green, Oscillating convection in an elasticoviscous liquid, The Physics of Fluids 11, 1410 (1968) https://aip.scitation.org/doi/pdf/10.1063/1.1692123

[20] C. M. Vest and V. S. Arpaci, Overstability of a viscoelastic fluid layer heated from below, Journal of Fluid Mechanics 36, 613 (1969)

[21] M. Sokolov and R. I. Tanner, Convective stability of a general viscoelastic fluid heated from below, The Physics of Fluids 15, 534 (1972), https://aip.scitation.org/doi/pdf/10.1063/1.1693945

[22] E. N. Krapivina and T. P. Lyubimova, Nonlinear regimes of convection of an elasticoviscous fluid in a closed cavity heated from below, Fluid Dynamics 35, 473 (2000)

[23] H. M. Park, K. S. Shin, and H. S. Sohn, Numerical simulation of thermal convection of viscoelastic fluids using the grid-by-grid inversion method, International Journal of Heat and Mass Transfer 52, 4851 (2009)

[24] H. M. Park and J. Y. Lim, A new numerical algorithm for viscoelastic fluid flows : The grid-by-grid inversion method, Journal of Non-Newtonian Fluid Mechanics 165, 238 (2010).

[25] K. V. Kovalevskaya and T. P. Lyubimova, Onset and nonlinear regimes of convection of an elastoviscous fluid in a closed cavity heated from below, Fluid Dynamics 46, 854 (2011).

[26] H. M. Park, Peculiarity in the rayleigh-bénard convection of viscoelastic fluids, International Journal of Thermal Sciences 132, 34 (2018).

[27] M. Lappa and A. Boaro, Rayleigh-bénard convection in viscoelastic liquid bridges, Journal of Fluid Mechanics 904, A2 (2020)

[28] I. A. Eltayeb, Nonlinear thermal convection in an elastico-viscous layer heated from below, Proceedings of the Royal Society of London. A. Mathematical and Physical Sciences 356, 161 (1977), https://royalsocietypublishing.org/doi/pdf/10.1098/rspa.1977.0127

[29] S. Rosenblat, Thermal convection in a viscoelastic liquid, Journal of Non-Newtonian Fluid Mechanics 21, 201 (1986)

[30] J. Martínez-Mardones and C. Pérez-García, Bifurcation analysis and amplitude equations for viscoelastic convective fluids, Il Nuovo Cimento D 14, 961 (1992)

[31] R. E. Khayat, Chaos and overstability in the thermal convection of viscoelastic fluids, Journal of Non-Newtonian Fluid Mechanics 53, 227 (1994)

[32] R. E. Khayat, Non-linear overstability in the thermal convection of viscoelastic fluids, Journal of NonNewtonian Fluid Mechanics 58, 331 (1995)

[33] J. Martínez-Mardones, R. Tiemann, D. Walgraef, and W. Zeller, Amplitude equations and pattern selection in viscoelastic convection, Phys. Rev. E 54, 1478 (1996)

[34] H. M. Park and H. S. Lee, Hopf bifurcations of viscoelastic fluids heated from below, Journal of Non-Newtonian Fluid Mechanics 66, 1 (1996)

[35] P. Parmentier, G. Lebon, and V. Regnier, Weakly nonlinear analysis of bénard-marangoni instability in viscoelastic fluids, Journal of Non-Newtonian Fluid Mechanics 89, 63 (2000)

[36] Z. Li and R. E. Khayat, Finite-amplitude rayleigh-bénard convection and pattern selection for viscoelastic fluids, Journal of Fluid Mechanics 529, 221-251 (2005)

[37] M. Lappa, Review: Possible strategies for the control and stabilization of marangoni flow in laterally heated floating zones, Fluid Dynamics \& Materials Processing 1, $171(2005)$.

[38] R. Savino and M. Lappa, Assessment of thermovibrational theory: Application to $\mathrm{g}$-jitter on the space station, Journal of Spacecraft and Rockets 40, 201 (2003). https://doi.org/10.2514/2.3954.

[39] M. Lappa, The patterning behaviour and accumulation of spherical particles in a vibrated nonisothermal liquid, Physics of Fluids 26, 093301 (2014). https://doi.org/10.1063/1.4893078

[40] M. Lappa, Numerical study into the morphology and formation mechanisms of threedimensional particle structures in vibrated cylindrical cavities with various heating conditions, Physical Review Fluids 1, 064203 (2016)

[41] M. Lappa, On the multiplicity and symmetry of particle attractors in confined non-isothermal fluids subjected to inclined vibrations, International Journal of Multiphase Flow 93, 71 (2017).

[42] M. Lappa, On the formation and morphology of coherent particulate structures in non-isothermal enclosures subjected to rotating g-jitters, Physics of Fluids 31, 073303 (2019), https://doi.org/10.1063/1.5098438

[43] M. Lappa and T. Burel, Symmetry breaking phenomena in thermovibrationally driven particle accumulation structures, Physics of Fluids in the press (2020).

[44] M. Lappa, Thermal Convection: Patterns, Evolution and 
Stability, Vol. 30 (John Wiley \& Sons, Ltd (2009, Chichester, England), 2009).

[45] M. Lappa, Rotating Thermal Flows in Natural and Industrial Processes (John Wiley \& Sons, Ltd, 2012).

[46] F. Mokhtari, S. Kaddeche, D. Henry, S. Bouarab, A. Medelfef, and V. Botton, Three-dimensional effect of high frequency vibration on convection in silicon melt, Phys. Rev. Fluids 5, 123501 (2020).

[47] J. Alexander, Low-gravity experiment sensitivity to residual acceleration - a review, Microgravity Science and Technology 3, 52 (1990).

[48] J. I. D. Alexander, S. Amiroudine, J. Ouazzani, and F. Rosenberger, Analysis of the low gravity tolerance of bridgman-stockbarger crystal growth ii. transient and periodic accelerations, Journal of Crystal Growth 113, 21 (1991)

[49] J. I. D. Alexander, J. P. Garandet, J. J. Favier, and A. Lizée, g-jitter effects on segregation during directional solidification of tin-bismuth in the mephisto furnace facility, Journal of Crystal Growth 178, 657 (1997).

[50] A. I. Feonychev and G. A. Dolgikh, Influence of vibration on heat and mass transfer in microgravity conditions, Microgravity Quarterly 4, 233 (1994).

[51] G. Z. Gershuni and A. V. Lyubimov, Thermal Vibrational Convection, Vol. -1 (Wiley, England, 1998).

[52] R. J. Naumann, An analytical model for transport from quasi-steady and periodic accelerations on spacecraft, International Journal of Heat and Mass Transfer 43, 2917 (2000)

[53] T. P. Lyubimova, A. V. Perminov, and M. Kazimardanov, Stability of quasi-equilibrium states and supercritical regimes of thermal vibrational convection of a williamson fluid in zero gravity conditions, International Journal of Heat and Mass Transfer 129, 406 (2019)

[54] B. Maryshev, T. P. Lyubimova, and D. Lyubimov, Two-dimensional thermal convection in porous enclosure subjected to the horizontal seepage and gravity modulation, Physics of Fluids 25, 084105 (2013). https://doi.org/10.1063/1.4817375

[55] I. B. Simonenko, A justification of the averaging method for a problem of convection in a field of rapidly oscillating forces and for other parabolic equations, Mathematics of the USSR-Sbornik 16, 245 (1972).

[56] G. Z. Gershuni and E. M. Zhukhovitskii, Free thermal convection in a vibrational field under conditions of weightlessness, Soviet Physics Doklady 24, 894 (1979).

[57] S. Zen'kovskaya and I. B. Simonenko, Effect of high frequency vibration on convection initiation, Fluid Dynamics - FLUID DYN 1, 35 (1966).

[58] G. Z. Gershuni, E. M. Zhukhovitskii, and Y. S. Yurkov, Vibrational thermal convection in a rectangular cavity, Fluid Dynamics 17, 565 (1982).

[59] G. Z. Gershuni and E. M. Zhukhovitskii, Vibrational thermal convection in zero gravity, Fluid. Mech. Sov. Res. 15 (1986).

[60] H. Khallouf, G. Z. Gershuni, and A. Mojtabi, Numerical study of two-dimensional thermovibrational convection in rectangular cavities, Numerical Heat Transfer, Part A: Applications 27, 297 (1995) https://doi.org/10.1080/10407789508913701

[61] S. Bouarab, F. Mokhtari, S. Kaddeche, D. Henry, V. Botton, and A. Medelfef, Theoretical and numerical study on high frequency vibrational convection: Influence of the vibration direction on the flow structure, Physics of Fluids 31, 043605 (2019) https://doi.org/10.1063/1.5090264

[62] R. Monti, D. Langbein, and J. J. Favier, Influence of residual accelerations on fluid physics and materials science experiments, in Fluid Sciences and Materials Science in Space: A European Perspective, edited by H. U. Walter (Springer Berlin Heidelberg, Berlin, Heidelberg, 1987) pp. 637-680.

[63] R. Monti, R. Savino, and M. Lappa, Microgravity sensitivity of typical fluid physics experiment, in The 17 th Microgravity Measurements Group Meeting, Cleveland, Ohio, 24-26 March 1998, Vol. 23 (1998) pp. 1-15, 17th International Microgravity Measurements Group (MGMG) meeting , 1998, MGMG 1998 ; Conference date: 24-031998 Through 26-03-1998.

[64] R. V. Birikh, V. A. Briskman, V. I. Chernatynski, and B. Roux, Control of thermocapillary convection in a liquid bridge by high frequency vibrations, Microgravity $Q$ 3, 23 (1993).

[65] K. Hirata, T. Sasaki, and H. Tanigawa, Vibrational effects on convection in a square cavity at zero gravity, Journal of Fluid Mechanics 445, 327 (2001)

[66] G. Crewdson and M. Lappa, The zoo of modes of convection in liquids vibrated along the direction of the temperature gradient, Fluids 6, 10.3390/fluids6010030 (2021).

[67] T. Lyubimova and K. Kovalevskaya, Gravity modulation effect on the onset of thermal buoyancy convection in a horizontal layer of the oldroyd fluid, Fluid Dynamics Research 48, 061419 (2016).

[68] A. Boaro and M. Lappa, Multicellular states of viscoelastic thermovibrational convection in a square cavity, Physics of Fluids 33, 033105 (2021). https://doi.org/10.1063/5.0041226

[69] M. Lappa and H. Ferialdi, Multiple solutions, oscillons, and strange attractors in thermoviscoelastic marangoni convection, Physics of Fluids 30, 104104 (2018), https://doi.org/10.1063/1.5040562

[70] J. Martinez-Mardones and C. Perez-Garcia, Linear instability in viscoelastic fluid convection, Journal of Physics: Condensed Matter 2, 1281 (1990)

[71] F. H. Harlow and J. E. Welch, Numerical calculation of time-dependent viscous incompressible flow of fluid with free surface, The Physics of Fluids 8, 2182 (1965), https://aip.scitation.org/doi/pdf/10.1063/1.1761178

[72] A. J. Chorin, Numerical solution of the navier-stokes equations, Mathematics of Computation 22, 745 (1968).

[73] R. Temam, Une méthode d'approximation de la solution des équations de navier-stokes, Bulletin de la Société Mathématique de France 96, 115 (1968)

[74] D. L. Brown, R. Cortez, and M. L. Minion, Accurate projection methods for the incompressible navier-stokes equations, Journal of Computational Physics 168, 464 (2001)

[75] N. A. Petersson, Stability of pressure boundary conditions for stokes and navier-stokes equations, Journal of Computational Physics 172, 40 (2001)

[76] S. Armfield and R. Street, An analysis and comparison of the time accuracy of fractional-step methods for the navier-stokes equations on staggered grids, International Journal for Numerical Methods in Fluids 38, 255 (2002) https://onlinelibrary.wiley.com/doi/pdf/10.1002/fld.217

[77] J. L. Guermond, P. Minev, and J. Shen, An overview of projection methods for incompressible flows, Computer Methods in Applied Mechanics and Engineering 195, 
$6011(2006)$

[78] C. M. Rhie and W. L. Chow, Numerical study of the turbulent flow past an airfoil with trailing edge separation, AIAA Journal 21, 1525 (1983) https://doi.org/10.2514/3.8284

[79] L. Moukalled F., Mangani and M. Darwish, The Finite Volume Method in Computational Fluid Dynamics: An Advanced Introduction with OpenFOAM@ and Matlabß, Vol. 113 (Springer, 2015) pp. XXIII, 791.

[80] J. L. Favero, A. R. Secchi, N. S. M. Cardozo, and H. Jasak, Viscoelastic flow analysis using the software openfoam and differential constitutive equations, Journal of Non-Newtonian Fluid Mechanics 165, 1625 (2010)

[81] R. Guénette and M. Fortin, A new mixed finite element method for computing viscoelastic flows, Journal of NonNewtonian Fluid Mechanics 60, 27 (1995)

[82] G. Lebon, P. Parmentier, O. Teller, and P. C. Dauby, Bénard-marangoni instability in a viscoelastic jeffreys' fluid layer, Rheologica Acta 33, 257 (1994).

[83] H. M. Park and D. H. Ryu, Hopf bifurcation in thermal convection of viscoelastic fluids within finite domains, Journal of Non-Newtonian Fluid Mechanics 101, 1 (2001)

[84] J. L. Rogers, M. F. Schatz, O. Brausch, and W. Pesch,
Superlattice patterns in vertically oscillated rayleighbénard convection, Phys. Rev. Lett. 85, 4281 (2000).

[85] J. L. Rogers, M. F. Schatz, J. L. Bougie, and J. B. Swift, Rayleigh-bénard convection in a vertically oscillated fluid layer, Phys. Rev. Lett. 84, 87 (2000).

[86] J. L. Rogers, W. Pesch, and M. F. Schatz, Pattern formation in vertically oscillated convection, Nonlinearity 16, C1 (2002).

[87] J. L. Rogers, W. Pesch, O. Brausch, and M. F. Schatz, Complex-ordered patterns in shaken convection, Phys. Rev. E 71, 066214 (2005).

[88] R. M. Clever and F. H. Busse, Steady and oscillatory bimodal convection, Journal of Fluid Mechanics 271, 103-118 (1994)

[89] A. Morozov and S. E. Spagnolie, Introduction to complex fluids, in Complex Fluids in Biological Systems: Experiment, Theory, and Computation, edited by S. E. Spagnolie (Springer New York, New York, NY, 2015) pp. 3-52.

[90] L. M. Pismen and B. Y. Rubinstein, Quasicrystalline and dynamic planforms in nonlinear optics, Chaos, Solitons and Fractals 10, 761 (1999).

[91] R. Lifshitz, Theory of color symmetry for periodic and quasiperiodic crystals, Rev. Mod. Phys. 69, 1181 (1997) 